\title{
Efficacy and safety of pharmacological interventions in second- or later-line treatment of patients with advanced soft tissue sarcoma: a systematic review
}

\author{
Sheetal Sharma', Shweta Takyarr', Stephanie C Mansonn ${ }^{2 *}$, Sarah Powell ${ }^{2}$ and Nicolas Penel ${ }^{3}$
}

\begin{abstract}
Background: Current guidelines recommend anthracycline-based chemotherapy primarily with doxorubicin either as monotherapy or in combination with ifosfamide as the first-line treatment for most advanced STS subtypes. Therapeutic options after failure of doxorubicin and/or ifosfamide are limited. This study aimed to comprehensively review available data on the activity and safety of interventions in second- or later-line treatment of advanced STS.

Methods: Electronic literature databases (Embase ${ }^{\oplus}$, MEDLINE ${ }^{\oplus}$, MEDLINE ${ }^{\circledast}$ In-Process, Cochrane Central Register of Controlled Trials, and Cochrane Database of Systematic Reviews) were searched from 1980 to 01 March 2012 to identify randomised controlled trials (RCTs) and non-randomised studies (both prospective and retrospective) evaluating pharmacological interventions in patients with advanced STS pre-treated with anthracycline- and/or ifosfamide-based therapy.
\end{abstract}

Results: The review identified six RCTs (one phase III and five phase II trials) and 94 non-randomised studies. Based on the primary trial endpoints, RCTs demonstrated favourable efficacy for pazopanib over placebo (PFS: 4.6 months vs. 1.6 months), gemcitabine plus dacarbazine over dacarbazine monotherapy (3-month PFS rate: $54.2 \%$ vs. 35.2\%), and trabectedin 3-weekly schedule over weekly schedule (TTP: 3.7 months vs. 2.3 months. The non-randomised studies demonstrated heterogeneity in efficacy and safety results.

Conclusions: Across the RCTs, pazopanib over placebo, gemcitabine-dacarbazine over dacarbazine, and trabectedin 3-weekly over weekly regimen clearly demonstrated a PFS advantage in the second- and later-line treatment of advanced STS. With only one phase III trial in this setting, there is a clear need for additional comparative trials to better understand the risk: benefit ratios of available agents and combinations.

Keywords: Systematic review, Pazopanib, Soft tissue sarcoma

\section{Background}

Soft tissue sarcomas (STS) are a heterogeneous group of rare tumours that arise predominantly from the embryonic mesoderm [1]. STS has more than 50 distinct histological subtypes, with leiomyosarcoma, liposarcoma, synovial sarcoma, undifferentiated pleomorphic sarcoma, and malignant peripheral nerve sheath tumours being among the most common subtypes [1]. STS occurs rarely and accounts for approximately $1 \%$ of malignancies in adults and

\footnotetext{
* Correspondence: stephanie.c.manson@gsk.com

${ }^{2}$ GlaxoSmithKline, Uxbridge, UK

Full list of author information is available at the end of the article
}

$2 \%$ of cancer mortality $[2,3]$. Nearly half of the patients diagnosed with STS develop advanced/metastatic disease and eventually die from the disease [4]. Patients typically demonstrate a median survival ranging from 11 to 18 months from diagnosis of advanced disease $[5,6]$.

The treatment for STS is largely dictated by the tumour grade, size, location of metastatic sites, and the histological subtype $[4,6,7]$. Outside of clinical trials, cytotoxic chemotherapy is the only available systemic therapy for patients with advanced disease and its goal is primarily palliative [6]. Current guidelines including the European Society for Medical Oncology and the National Comprehensive Cancer

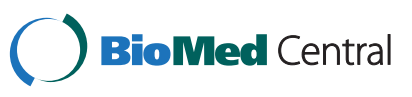


Network treatment guidelines recommend anthracyclinebased chemotherapy - primarily with doxorubicin, either as monotherapy or in combination with ifosfamide, as the first-line treatment for most advanced STS subtypes $[7,8]$. Therapeutic options after failure of doxorubicin and/or ifosfamide are limited and there are no standard recognised therapies. Options used in clinical practice include ifosfamide, trabectedin, gemcitabine in combination with docetaxel, and dacarbazine-based regimens [9]. With the advent of new targeted therapies for treatment of advanced STS, it is important to understand the current evidence base in this setting. We aimed to comprehensively review available data on the efficacy and safety of treatments used for patients with advanced STS pre-treated with anthracycline- and/or ifosfamidebased therapy. The comparability amongst this evidence was examined in light of recent Phase III trial evidence for pazopanib, a new oral selective tyrosine kinase inhibitor for the treatment of advanced STS.

\section{Methods}

In order to provide a robust assessment of the available evidence, a systematic review was undertaken to identify, describe and interpret the current state of evidence. The review was conducted in accordance with the Preferred Reporting Items for Systematic Reviews and Meta-Analyses (PRISMA) guidelines (See Additional file 1) [10].

\section{Searching}

The review was based on a comprehensive search of MEDLINE $^{\oplus}$, including MEDLINE ${ }^{\oplus}$ In-Process, Embase ${ }^{\oplus}$, Cochrane Central Register of Controlled Trials (CENTRAL), and Cochrane Database of Systematic Reviews from 1980 to 01 March 2012. An additional file describes the search strategy used for MEDLINE ${ }^{\bullet}$ and Embase ${ }^{\oplus}$ (see Additional file 2).

In addition to the literature database search, abstracts from conference proceedings including American Society of Clinical Oncology, European Society for Medical Oncology, European Conference for Clinical Oncology, Connective Tissue Oncology Society and Musculoskeletal Tumour Society were hand searched from 2007 to March 2012. For trials in progress, Clinicaltrials.gov, UK clinical trials gateway, and International Standard Randomised Controlled Trial Number were searched. Bibliographic searching of included trials and systematic reviews was also performed.

\section{Study selection and characteristics}

The review included randomised controlled trials (RCTs) and non-randomised studies (prospective and retrospective studies) in patients with pre-treated advanced STS. The review was limited to studies in which patients had received prior anthracycline and/or ifosfamide therapy since these are generally considered to be the standard of care for the first-line treatment of advanced STS [7-9]. References were excluded from the review if they recruited paediatric patients $(<18$ years old). Studies exclusively enrolling patients with gastrointestinal stromal tumours (GIST), Kaposi sarcoma, and Ewing's family of tumours were also excluded because of their unique biology and management compared with other STS subtypes. Studies that recruited a mixed STS population including GIST, Kaposi sarcoma, or Ewing tumours with no appropriate subgroup data by histological subtype were excluded. Other exclusion criteria were where no subgroup data for patients with advanced stage STS were available across trials recruiting both patients with early stage and advanced STS, or where studies included a mixed population of treatment-naive patients and patients previously treated with anthracycline- and/or ifosfamide-based therapy with no subgroup data for the pre-treated patients.

Further, to be included in the review, studies were required to be published in English and investigating a therapy identified either in STS treatment guidelines $[6-8,11]$, cited in STS treatment review papers $[2,12,13]$ or being researched in the pre-treated advanced STS setting (carboplatin, cyclophosphamide, dacarbazine, docetaxel, doxorubicin, epirubicin, etoposide, gemcitabine, ifosfamide, liposomal doxorubicin, paclitaxel, pazopanib, trabectedin, vincristine, cisplatin, vinblastine, methotrexate, tamoxifen, sunitinib, sorafenib, deforolimus, temsirolimus, everolimus, gefitinib, erlotinib, cetuximab, or brostallicin alone or in combination). Comparative studies were included if the intervention of interest was compared with placebo, best supportive care, or any of the included interventions.

All studies retrieved by searches were screened according to above defined eligibility criteria. Initial screening of the retrieved citations was conducted independently by two reviewers on the basis of the title and abstract. Any discrepancies between the reviewers were resolved by a third independent reviewer. The full-text publications of all citations of potential interest were then screened for inclusion by two reviewers (SS and ST), with disagreements resolved by a third independent reviewer.

\section{Validity assessment}

Quality assessment of RCTs was performed using a comprehensive critical appraisal tool based on the National Institute for Health and Clinical Excellence's and Cochrane's critical appraisal tool $[14,15]$. Critical appraisal of comparative studies (other than RCTs) and single-arm studies was conducted using the Downs and Black checklist [16]. The data endpoints extracted included overall survival (OS), progression-free survival (PFS), overall response rate (ORR), complete response (CR), partial response $(\mathrm{PR})$, stable disease $(\mathrm{SD})$, progressive disease $(\mathrm{PD})$, time 
to progression (TTP), duration of response (DOR), time to response (TTR), EORTC Quality of Life-QuestionnaireC30 score, EQ-5D score, adverse events, and withdrawals.

\section{Data abstraction}

Relevant data from all included studies were extracted using a pre-defined extraction grid. Data extraction was conducted in parallel by two independent reviewers with any differences resolved by a third independent reviewer. Where more than one publication was identified that described a single trial, the data were compiled into a single entry to avoid double counting of patients.

\section{Quantitative data synthesis}

There was considerable heterogeneity across studies in terms of interventions, comparisons, patient population, and study designs. Further, the available evidence base was limited with no more than one study directly comparing the same set of interventions. Therefore metaanalysis, indirect, and mixed treatment comparison of the included interventions were not appropriate. We describe the results qualitatively with detailed results presented in supporting tables. The results are presented separately for RCTs and non-randomised studies.

\section{Results}

\section{Trial flow / flow of included studies}

The flow of studies through the review, according to PRISMA guidelines [17], is shown in Figure 1.

The search of the literature databases yielded 9542 separate references. Following the screening of abstracts against the inclusion/exclusion criteria, 545 full-text

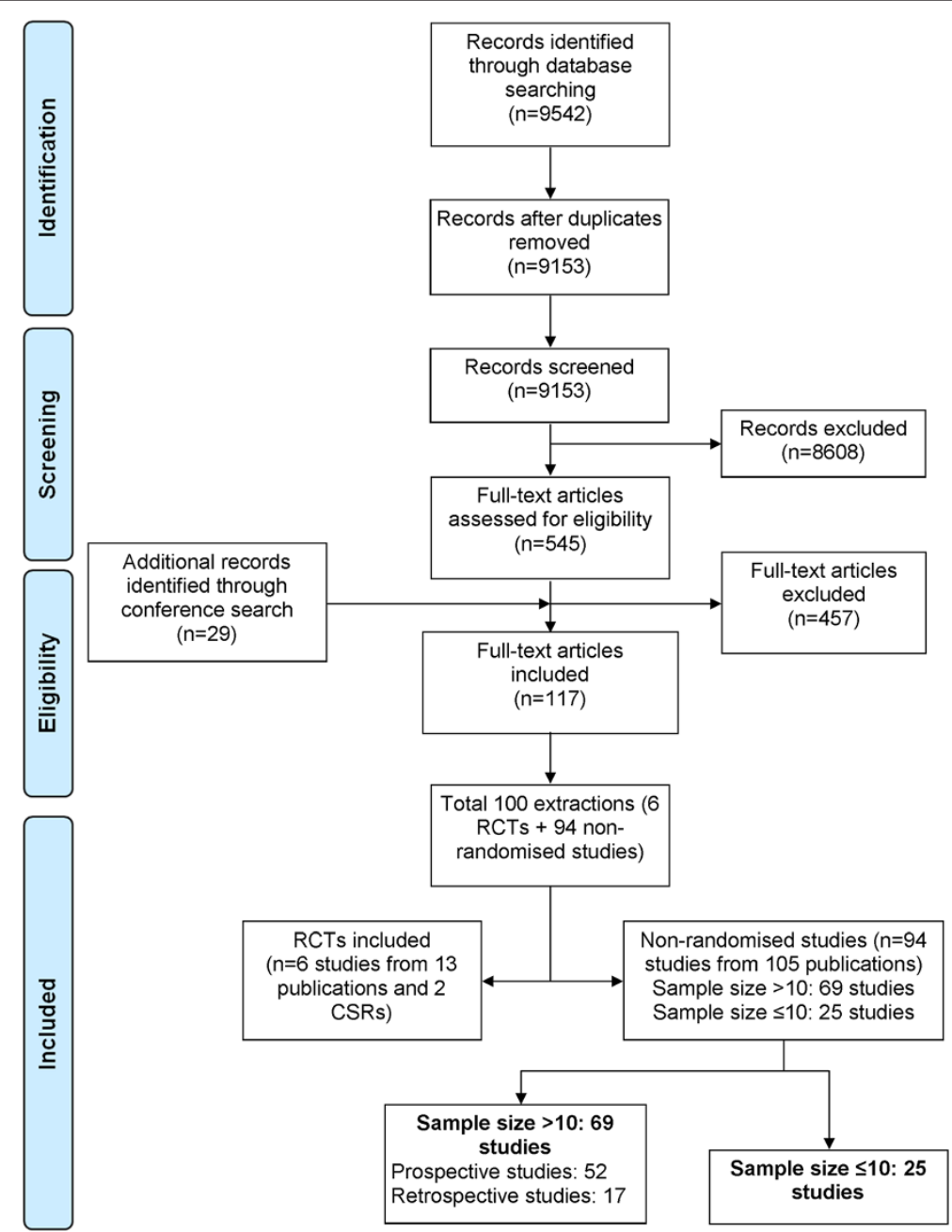

Figure 1 Flow of studies through the systematic review process. The figure describes the flow of studies through the review, according to PRISMA guidelines. The search of the literature databases yielded 9542 separate references. Following the screening of abstracts and full-text publications against the inclusion/exclusion criteria a total of six RCTs (reported in 13 publications) and 94 non-randomised studies (reported in 105 publications) met the inclusion criteria for the review. 
reports were obtained for detailed evaluation. Additionally, 29 references meeting the inclusion/exclusion criteria for the review were identified from conference proceedings. After screening, a total of six RCTs (reported in 13 publications and two Clinical Study Reports) and 94 non-randomised studies (reported in 105 publications) met the inclusion criteria for the review. The list of the 457 studies excluded from the review along with exclusion rationale is available on request.

RCTs

\section{Study characteristics}

The key patient and study design characteristics of the six included RCTs are presented in Table 1. All included RCTs were Phase II trials, except for the PALETTE study, which was the only Phase III RCT [18,19]. All the studies included in the review aimed to evaluate the activity and safety of the interventions under investigation, with PFS being the primary outcome in two studies [18-21] and TTP [22-26], response [27], and 12-week progressionfree rate [28], as the primary outcomes in one study each. There was no primary endpoint identified in the remaining one study [29]. The secondary outcome measures evaluated across these studies included OS, response, DOR, TTR, dose reductions/interruptions, safety, and withdrawals.

The number of patients randomised across all RCTs was greater than 50, except for the study by Pacey and colleagues that randomised five patients [28]. Of the five patients randomised in this study, one patient was chemotherapy-naive and hence did not meet the inclusion criteria of the review. In addition, this small-sized study was not a true RCT [28]. All the patients initially received sorafenib in a 12-week open-label run-in period following which patients with $\geq 25 \%$ tumour shrinkage continued sorafenib, those with $\geq 25 \%$ tumour growth discontinued, and the remaining patients were randomised to treatment with sorafenib (2 patients) or placebo (2 patients) [28]. In terms of the patient population recruited across these studies, leiomyoscaroma was the most commonly enrolled subtype of STS followed by liposarcoma and undifferentiated pleomorphic sarcoma. Across all the included RCTs, at least $90 \%$ of patients received prior treatment in an advanced setting, except for the study by van Oosterom and colleagues [29]. This study recruited a mixed population of patients previously treated in an adjuvant or advanced setting, with limited subgroup data for the patients previously treated in the advanced setting [29].

The quality assessment of the included RCTs using the comprehensive critical appraisal tool based on the NICE and Cochrane's critical appraisal tool is detailed in an additional file (see Additional file 3). None of the RCTs included in the review were identified as being at a high risk of bias.

\section{Efficacy/activity results}

Table 2 summarises the various efficacy/activity results observed across the included RCTs. The RCTs included in the review have been examined separately according to the phase of the trial.

\section{Phase III trials}

The only Phase III trial included in the review was the PALETTE trial evaluating pazopanib $(\mathrm{N}=246)$ versus placebo $(\mathrm{N}=123)$ in advanced STS patients (excluding GIST, liposarcoma and other subtypes). The data presented here are from an analysis conducted by the manufacturer for regulatory purposes $[18,19]$ and differ slightly from an analysis conducted by the study's collaborative partner $[30,32]$ as a consequence of small differences in censoring rules and data handling. A summary of these minor differences in results between analyses can be found in Additional file 4. This trial demonstrated a significantly prolonged primary endpoint of PFS (per independent review) for pazopanib compared with placebo (Hazard Ratio (HR): 0.35 [95\% CI: 0.26 - 0.48]; $\mathrm{p}<0.001)[18,19]$. The benefit in PFS was consistently observed across all three histological sub-types included in the study (leiomyosarcoma [ $<<0.001]$, synovial sarcoma $[\mathrm{p}=0.005]$, and other STS sub-types $[\mathrm{p}<0.001])$. The best overall response based on the independent radiology review also favoured pazopanib. However, there was no statistically significant difference between pazopanib and placebo for median OS (HR: 0.87 [95\% CI: 0.67 - 1.12]; $\mathrm{p}=0.256)[18,19]$. These results should be interpreted in view of the fact that patients treated with pazopanib and placebo received post-study therapy including trabectedin ( $25 \%$ vs. $32 \%)$, gemcitabine ( $17 \%$ vs. $23 \%)$, a taxane $(10 \%$ vs. $18 \%)$ and ifosfamide (10\% vs. $17 \%)$ that might have potentially confounded the OS results [19]. This was the only study to report quality of life data. Based on the EORTC QLQ-C30 questionnaire, no clinically meaningful or statistically significant differences in global health status were observed between pazopanib and placebo patients remaining on treatment at the assessment time points $[18,19]$.

\section{Phase II trials}

PFS rate at 3 months was the primary activity measure in the GEIS study comparing the combination of gemcitabine and dacarbazine $(\mathrm{N}=59)$ against dacarbazine monotherapy $(\mathrm{N}=54)[20,21]$. The PFS rate at 3 months was significantly better for gemcitabine plus dacarbazine than dacarbazine monotherapy $(\mathrm{p}=0.001)$. Similar results, favouring the combination, were observed in terms of the secondary efficacy endpoints evaluated including median PFS (HR: 0.58 [95\% CI: 0.39 - 0.86]; $\mathrm{p}=0.005$ ), median OS (HR: 0.56 [95\% CI: $0.36-0.90] ; \mathrm{p}=0.014)$, and response rate $[20,21]$. Fifty-three percent of patients initially treated 
Table 1 Summary of relevant randomised controlled trials included in the review

\begin{tabular}{|c|c|c|c|c|c|c|c|c|c|}
\hline Interventions & Study & Study design & $\mathrm{N}^{*}$ & $\begin{array}{l}\text { Age median } \\
\text { (range) }\end{array}$ & $\begin{array}{l}\text { Males } \\
(\%)\end{array}$ & $\begin{array}{l}\text { Median duration } \\
\text { of follow-up } \\
\text { (weeks) }\end{array}$ & $\begin{array}{l}\text { Prior therapy for } \\
\text { advanced disease }\end{array}$ & $\begin{array}{l}\text { Performance status, } \\
\text { n (\%) }\end{array}$ & STS subtypes, (\%) \\
\hline $\begin{array}{l}\text { Pazopanib } 800 \text { mg per } \\
\text { day orally }\end{array}$ & $\begin{array}{l}\text { PALETTE study } \\
2011[18,19,30]\end{array}$ & $\begin{array}{l}\text { R, DB, PC, MC-I, } \\
\text { Phase III }\end{array}$ & 246 & $56.0(20.0-83.0)$ & $40.0 \%$ & 49.5 weeks & $\begin{array}{l}\text { Anthracycline: } 98.8 \% \text {; } \\
\text { ifosfamide: } 66.7 \%\end{array}$ & $\begin{array}{l}\text { PS 0: } 118(48.0) ; \\
\text { PS 1: } 128(52.0)\end{array}$ & $\begin{array}{l}\text { Leiomyosarcoma: } 44.3 \% \text {; } \\
\text { Synovial sarcoma: } 10.2 \% \text {; } \\
\text { Others: } 45.5 \%\end{array}$ \\
\hline Placebo & $\begin{array}{l}\text { PALETTE study } \\
2011[18,19,30]\end{array}$ & & 123 & $51.0(18.0-78.0)$ & $44.0 \%$ & 45.3 weeks & $\begin{array}{l}\text { Anthracycline: } 98.4 \% \text {; } \\
\text { ifosfamide: } 75.6 \%\end{array}$ & $\begin{array}{l}\text { PS 0: } 60 \text { (48.8); } \\
\text { PS 1: } 67(51.2)\end{array}$ & $\begin{array}{l}\text { Leiomyosarcoma: } 39.8 \% \text {; } \\
\text { Synovial sarcoma: } 10.6 \% \text {; } \\
\text { Others: } 49.6 \%\end{array}$ \\
\hline $\begin{array}{l}\text { Trabectedin } 1.5 \mathrm{mg} / \mathrm{m} 2 \\
\text { 24-hour infusion q3w }\end{array}$ & $\begin{array}{l}\text { Demetri } 2009 \\
\text { [22-26] }\end{array}$ & $\begin{array}{l}\text { R, OL, DR, MC-I, } \\
\text { Phase II }\end{array}$ & 136 & $53(20-80)$ & $32.4 \%$ & 177.67 weeks & $\begin{array}{l}\text { Anthracycline: } 100 \% \text {; } \\
\text { anthracycline and } \\
\text { ifosfamide: } 99.3 \%\end{array}$ & $\begin{array}{l}\text { PS 0: } 70 \text { (51.5); } \\
\text { PS 1: } 66 \text { (48.5) }\end{array}$ & $\begin{array}{l}\text { Leiomyosarcoma: } 61.5 \% \text {; } \\
\text { Liposarcoma: } 25.6 \% \text {; } \\
\text { Others: } 12.8 \%\end{array}$ \\
\hline $\begin{array}{l}\text { Trabectedin } 0.58 \text { mg/m2 } \\
\text { 3-hour infusion qw }\end{array}$ & $\begin{array}{l}\text { Demetri } 2009 \\
\text { [22-26] }\end{array}$ & & 134 & $54(23-77)$ & $41.8 \%$ & & & $\begin{array}{l}\text { PS 0: } 67(50.0) ; \\
\text { PS 1: } 67(50.0)\end{array}$ & $\begin{array}{l}\text { Leiomyosarcoma: } 55.5 \% \text {; } \\
\text { Liposarcoma: } 37.8 \% \text {; } \\
\text { Others: } 6.7 \%\end{array}$ \\
\hline $\begin{array}{l}\text { Gemcitabine } 1800 \mathrm{mg} / \mathrm{m} 2 \\
\text { as a fixed dose infusion rate } \\
\text { (10 mg/m2/minutes) + } \\
\text { dacarbazine } 500 \mathrm{mg} / \mathrm{m} 2 \mathrm{q} 2 \mathrm{w}\end{array}$ & $\begin{array}{l}\text { GEIS study } \\
{[20,21] \sim}\end{array}$ & $\begin{array}{l}\mathrm{R}, \mathrm{BU}, \mathrm{AC}, \mathrm{MC} \\
\text { Phase II }\end{array}$ & 59 & $49(18-78)$ & $53.0 \%$ & 62.83 weeks & $\begin{array}{l}\text { Out of total eligible } \\
\text { population of } 109 \\
\text { patients } 107 \text { patients } \\
\text { had received anthracycline } \\
\text { and two patients had } \\
\text { received ifosfamide }\end{array}$ & $\begin{array}{l}\text { PS 0:22 (38.6); } \\
\text { PS 1:30 (52.6); } \\
\text { PS 2:5 (8.8) }\end{array}$ & $\begin{array}{l}\text { Leiomyosarcoma: 28.1\%; } \\
\text { Liposarcoma/adipocytic } \\
\text { sarcoma: } 17.5 \% ; \\
\text { Undifferentiated } \\
\text { pleomorphic: } 19.3 \% ; \\
\text { Miscellaneous sarcoma: } 24.6 \% \text {; } \\
\text { Synovial sarcoma: } 10.5 \%\end{array}$ \\
\hline Dacarbazine 1200 mg/m2 q3w & $\begin{array}{l}\text { GEIS study } \\
{[20,21] \sim}\end{array}$ & & 54 & $51(25-73)$ & $54.0 \%$ & 60.67 weeks & & $\begin{array}{l}\text { PS 0: } 17(32.7) ; \\
\text { PS 1: } 31(51.6) ; \\
\text { PS 2: } 4(7.7)\end{array}$ & $\begin{array}{l}\text { Leiomyosarcoma: 30.8\%; } \\
\text { Liposarcoma/adipocytic } \\
\text { sarcoma: 17.3\%; } \\
\text { Undifferentiated pleomorphic: } \\
\text { 15.4\%; Miscellaneous sarcoma: } \\
\text { 26.9\%; Synovial sarcoma: 9.6\% }\end{array}$ \\
\hline $\begin{array}{l}\text { Gemcitabine } 900 \text { mg/m2 } \\
\text { over } 90 \text { minutes, D1+D8 + } \\
\text { docetaxel } 100 \text { mg/m2 over } \\
60 \text { min, D8 q21 days }\end{array}$ & $\begin{array}{l}\text { Pautier } 2009 \\
{[27,31] \sim}\end{array}$ & $\begin{array}{l}R, B U, A C, M C \\
\text { Phase II }\end{array}$ & $84^{* *}$ & - & - & Unclear & Anthracycline: 100\% & - & Leiomyosarcoma: 100\% \\
\hline $\begin{array}{l}\text { Gemcitabine } 1000 \mathrm{mg} / \mathrm{m} 2 \\
\text { over } 100 \text { minutes, } \mathrm{d} 1+\mathrm{d} 8+\mathrm{d} 15 \\
\text { q28 days }\end{array}$ & $\begin{array}{l}\text { Pautier } 2009 \\
{[27,31] \sim}\end{array}$ & & & - & - & & Anthracycline: 100\% & - & Leiomyosarcoma: 100\% \\
\hline $\begin{array}{l}\text { Sorafenib } 400 \mathrm{mg} \text { twice } \\
\text { daily orally }\end{array}$ & Pacey 2011 [28] & $\begin{array}{l}\text { R, DB, PC, MC-I, } \\
\text { Phase II }\end{array}$ & 2 & - & - & Unclear & $\begin{array}{l}\text { Anthracycline and/or } \\
\text { ifosfamide: } 100 \%\end{array}$ & - & Fibrosarcoma: 0.0\% \\
\hline \multirow[t]{2}{*}{ Placebo } & \multirow[t]{2}{*}{ Pacey 2011 [28] } & & 2 & $67(62-72)$ & 0 & & & PS 0: 2 (100) & \multirow{2}{*}{$\begin{array}{l}\text { Leiomyosarcoma: } 50.0 \% \text {; } \\
\text { Fibrosarcoma: } 50.0 \%\end{array}$} \\
\hline & & & & & & & & PS 1: $0(0.0)$ & \\
\hline $\begin{array}{l}\text { Ifosfamide } 5 \mathrm{~g} / \mathrm{m} 2 / 1 \text { day given } \\
\text { as } 24 \text {-hour infusion; all cycles } \\
\text { were repeated q3w }\end{array}$ & $\begin{array}{l}\text { van Oosterom } \\
2002[29]\end{array}$ & $\begin{array}{l}\text { R, BU, DR, MC-I, } \\
\text { Phase II }\end{array}$ & 27 & - & - & Unclear & Anthracycline: 100\% & - & - \\
\hline
\end{tabular}


Table 1 Summary of relevant randomised controlled trials included in the review (Continued)

Ifosfamide $3 \mathrm{~g} / \mathrm{m} 2 /$ day given van Oosterom

over 4 hour on 3 consecutive 2002 [29]

Anthracycline: 100\%

days; all cycles were

repeated q3w

AC: Active-controlled; BU: Blinding Unclear; DB: Double-blind; DR: Dose Ranging; ECOG: Eastern Cooperative Oncology Group; q3w: Every Three Weeks; q2w: Every Two weeks; qw: Every week; MFH: Malignant Fibrous Histiocytoma; min: Minutes; MC: Multicentre; MC-I: Multicentre International; OL: Open Label; PS: Performance Status; STS: Soft Tissue Sarcoma; *N represents number of patients randomised except for Pacey 2011

study and van Oosterom 2002 where $\mathrm{N}$ represents the patient population of interest with respect to prior treatment for advanced disease; **Represents total number of patients randomised in the study (number of

patients randomised to each arm not reported); - Represents data not reported; $\uparrow$ Represents data for the complete study population; Represents secondary reference. 
Table 2 Summary of various efficacy/activity outcomes observed across randomised controlled trials

\begin{tabular}{|c|c|c|c|c|c|c|c|c|c|c|c|c|}
\hline \multirow[t]{3}{*}{ Intervention } & \multirow[t]{3}{*}{ Study } & \multirow[t]{3}{*}{$N$} & \multicolumn{3}{|c|}{ Progression free survival } & \multicolumn{2}{|c|}{ Overall survival } & \multicolumn{4}{|c|}{ Response rate } & \multirow{3}{*}{$\begin{array}{l}\text { Progressive } \\
\text { disease } \\
\text { PD } \\
\text { n (\%) }\end{array}$} \\
\hline & & & \multicolumn{2}{|c|}{ PFS rate, $\mathrm{n}(\%)$} & \multirow{2}{*}{$\begin{array}{l}\text { PFS in months } \\
\text { median }(95 \% \mathrm{Cl})\end{array}$} & \multirow{2}{*}{$\begin{array}{l}1 \text { year OS } \\
\mathrm{n}(\%)\end{array}$} & \multirow{2}{*}{$\begin{array}{l}\text { OS in months } \\
\text { median }(95 \% \mathrm{Cl})\end{array}$} & \multirow{2}{*}{$\begin{array}{l}\text { ORR } \\
\text { n (\%) }\end{array}$} & \multirow{2}{*}{$\begin{array}{l}\text { CR } \\
\text { n (\%) }\end{array}$} & \multirow{2}{*}{$\begin{array}{l}\text { PR } \\
\text { n (\%) }\end{array}$} & \multirow{2}{*}{$\begin{array}{l}\text { SD } \\
\text { n (\%) }\end{array}$} & \\
\hline & & & 3-month & 6-month & & & & & & & & \\
\hline Pazopanib & PALETTE study 2011/2 & 246 & - & - & $4.6+$ & - & 12.6 & $11(4.5)+$ & $0(0.0)+$ & $11(4.5)+$ & $134(54.5) \dagger$ & $66(26.8)+$ \\
\hline Placebo & PALETTE study 2011 & 123 & - & - & $1.6+$ & - & 10.7 & $0(0.0)+$ & $0(0.0)+$ & $0(0.0)+$ & $33(26.8)+$ & $76(61.8)+$ \\
\hline Trabectedin $1.5 \mathrm{mg} / \mathrm{m} 2 \mathrm{q} 3 \mathrm{w}$ & Demetri 2009 & 136 & $70(51.5)$ & $48(35.5)$ & $3.3+(2.1-4.6)$ & $82(60.0)$ & $13.9(12.5-18.6)$ & $8(5.6) \dagger$ & - & - & - & - \\
\hline Trabectedin $0.58 \mathrm{mg} / \mathrm{m} 2 \mathrm{qw}$ & Demetri 2009 & 134 & $60(44.7)$ & $37(27.5)$ & $2.3+(2.0-3.4)$ & $67(50.0)$ & $11.8(9.9-14.9)$ & $2(1.6) \dagger$ & - & - & - & - \\
\hline Dacarbazine & GEIS study & 54 & $\begin{array}{l}19(35.2) ; \\
p=0.001\end{array}$ & - & 2.0\# & - & $8.2 \S$ & $\begin{array}{l}2(3.7) \neq, \\
p=0.16\end{array}$ & - & $2(4.0)^{*} \#$ & $10(19.0)^{*} \#$ & - \\
\hline Gemcitabine + Dacarbazine & GEIS study & 59 & $\begin{array}{l}32(54.2) \\
p=0.001\end{array}$ & - & 4.2\# & - & $16.8 \S$ & $\begin{array}{l}7 \text { (11.9)丮, } \\
\mathrm{p}=0.16\end{array}$ & - & $5(9.0)^{*} \#$ & $22(38.0)^{*} \#$ & - \\
\hline Sorafenib & Pacey 2011 & 2 & - & - & - & - & - & - & - & - & - & $0(0.0) \#$ \\
\hline Placebo & Pacey 2011 & 2 & - & - & - & - & - & - & - & $0(0.0) \#$ & $2(100) \#$ & $0(0.0) \#$ \\
\hline Gemcitabine & Pautier 2009 & - & - & - & - & - & - & - & - & - & - & - \\
\hline Gemcitabine + Docetaxel & Pautier 2009 & - & - & - & - & - & - & - & - & - & - & - \\
\hline Ifosfamide $5 \mathrm{~g} / \mathrm{m}^{2} /$ day & van Oosterom 2002 & 27 & - & - & - & - & - & - & - & - & - & - \\
\hline Ifosfamide $3 \mathrm{~g} / \mathrm{m}^{2} /$ day & van Oosterom 2002 & 31 & - & - & - & - & - & - & - & - & - & - \\
\hline
\end{tabular}

Cl: Confidence Interval; CR: Complete Response; INV: Investigator; IRC: Independent Review Committee; N: Number of evaluable Patients; $\mathrm{n}$ : Number with Outcome; ORR: Overall Response Rate; OS: Overall Survival; PFS: Progression-free Survival; PR: Partial Response; $q 3 w$ : Every Three Weeks; $q w$ : Every Week: SD: Stable Disease; * $p=0.01$; †Assessments were made by the independent review committee; $¥$ Assessments were made by the investigator; \#Unclear if assessed by investigator or the Independent Review Committee; SKaplan-Meier estimates reported; -Represents data not reported. 
with dacarbazine monotherapy and $51 \%$ of patients treated with the combination of gemcitabine and dacarbazine received post-study therapy comprising mainly gemcitabinebased regimens, trabectedin, and taxanes [20,21].

TTP was the primary activity endpoint in the study by Demetri and colleagues evaluating the two dosing schedules of trabectedin [22-26]. Median TTP favoured the trabectedin q3w 24-hour dosing schedule $(\mathrm{N}=136)$ over the qw 3-hour dosing schedule $(\mathrm{N}=134)$ when assessment was made by investigator (4.2 months vs. 2.5 months; HR: 0.668 [95\% CI: $0.506-0.883$ ]; $\mathrm{p}=0.0042$ ) and IRC (3.7 months vs. 2.3 months; HR: 0.734 [95\% CI: $0.554-$ 0.974 ]; $\mathrm{p}=0.03$ ) [22-26]. In terms of the secondary activity measures, median PFS was significantly longer with the q3w 24-hour schedule than the qw 3-hour schedule $(\mathrm{p}=0.0418)$, while no significant differences between the two dosing schedules were observed in median OS (HR: 0.843 [95\% CI: 0.653 - 1.090]; $\mathrm{p}=0.1920$ ) [22-26]. The PFS rate, 1-year OS rate, and ORR also favoured the q3w 24-hour dosing schedule over the qw 3-hour dosing schedule. Forty-nine patients in this study received poststudy therapy by crossing-over to the other schedule (35 patients crossed-over after progression as allowed by the protocol [29 patients from qw 3-hour arm to q3w 24-hour arm, and 6 patients from q3w 24-hour arm to qw 3-hour arm] and 14 patients before progression [following independent data monitoring committee recommendation, all from qw 3-hour to q3w 24-hour arm]) [22-26].

Limited activity data were obtained from the remaining three RCTs included in the review [27-29]. The study by van Oosterom and colleagues evaluating two different ifosfamide regimens provided no subgroup efficacy data including OS, TTP, PFS, and response duration specifically for patients previously treated in an advanced setting [29]. Similarly, in the study by Pacey and colleagues, although the PFS rate at 12 weeks was the primary activity endpoint, the study did not report data for patients randomised to sorafenib or placebo. The only activity data reported in this study was SD in all four patients receiving sorafenib or placebo at 12 weeks [28]. Activity data were also not reported in the conference abstract for the TAXOGEM study by Pautier and colleagues [27].

\section{Safety results}

Overall, AEs were not consistently reported across the RCTs included in the review. The most commonly reported grade $3 / 4$ AEs $(\geq 5 \%)$ in association with pazopanib in the Phase III PALETTE trial were fatigue (14\%), lymphopenia (10\%) tumour pain $(8 \%)$, increased alanine transaminase (ALT) (10\%), increased aspartate aminotransferase (AST) (8\%), hypertension (7\%), dyspnoea (6\%), anaemia (6\%), decreased appetite (6\%), and diarrhoea (5\%) $[18,19]$. Across the Phase II trials, haematological AEs were commonly experienced with treatments including dacarbazine, gemcitabine plus dacarbazine, and trabectedin. In addition to haematological AEs, $\geq 5 \%$ of patients experienced grade $3 / 4$ ALT increase, creatinine phosphokinase increase, and fatigue with the two dosing schedules of trabectedin [22-26] and grade 3/4 asthenia with gemcitabine plus dacarbazine and dacarbazine monotherapy [20,21]. Grade $3 / 4$ nausea, vomiting, and AST increase were also experienced by $\geq 5 \%$ of patients treated with trabectedin 24-hour schedule [22-26]. Summaries for grade 3 and/or 4 AEs reported in $>1 \%$ patients across the included studies are shown in Table 3.

\section{Treatment discontinuations}

Overall, four of the six included RCTs reported data related to treatment discontinuations. In the Phase III PALETTE trial, pazopanib was associated with a higher proportion of patients discontinuing treatment due to AEs compared with placebo (Table 4) [19]. Across the Phase II RCTs, the proportion of patients discontinuing treatment due to AEs were comparable with the two dosing schedules of trabectedin [22-26], while in the GEIS study none of the patients treated with gemcitabine plus dacarbazine discontinued therapy due to AEs [20,21]. Summaries of the treatment discontinuations observed across the included RCTs are shown in Table 4.

\section{Non-randomised studies \\ Trial characteristics}

A summary of the 52 prospective non-randomised studies with sample size more than 10 [33-72] is presented in Table 5. Further details regarding the study design and patient characteristics for these studies are presented in an additional file (see Additional file 5). The list of retrospective studies and studies with a sample size less than 10 is also provided as an additional file (see Additional file 6).

The majority of the included prospective studies were Phase II trials with a variety of chemotherapeutic regimens evaluated across these studies. Ifosfamide was the most commonly evaluated monotherapy (nine studies) followed by gemcitabine (four studies), docetaxe 1 (three studies), paclitaxel (three studies), and trabectedin (three studies), while gemcitabine-based regimens were the most frequently evaluated combination therapy (five studies). Response, PFS, DOR, TTP, OS, and safety were the most commonly assessed outcomes in the included studies.

The quality assessment of the included non-randomised studies based on the Downs and Black checklist demonstrated that studies were reported reasonably well in terms of study question, methods, patient population, outcomes measures, and results [16].

\section{Efficacy results}

Across the non-randomised evidence, there was heterogeneity in the efficacy results. For example, in nine studies 
Table 3 Summary of grade 3 and/or 4 specific adverse events reported in $>1 \%$ patients across randomised controlled trials

\begin{tabular}{|c|c|c|c|c|c|c|c|c|c|c|c|c|}
\hline \multirow[t]{2}{*}{ AEs by class } & \multicolumn{2}{|c|}{ PALETTE study $2011^{\#}$} & \multicolumn{2}{|l|}{ Demetri 2009} & \multicolumn{2}{|c|}{ GEIS study 2011} & \multicolumn{2}{|l|}{ Pautier 2009} & \multicolumn{2}{|c|}{ Pacey 2011* } & \multicolumn{2}{|c|}{ van Oosterom 2002} \\
\hline & $\begin{array}{l}\text { Pazopanib, } \\
\text { n (\%) }\end{array}$ & $\begin{array}{l}\text { Placebo, } \\
\text { n (\%) }\end{array}$ & $\begin{array}{l}\text { Trabectedin } \\
1.5 \mathrm{mg} / \mathrm{m} 2 \mathrm{q} 3 \mathrm{w}, \\
\mathrm{n}(\%)\end{array}$ & $\begin{array}{l}\text { Trabectedin } \\
0.58 \mathrm{mg} / \mathrm{m} 2 \mathrm{qw}, \\
\mathrm{n}(\%)\end{array}$ & $\begin{array}{l}\text { Dacarbazine, } \\
\text { n (\%) }\end{array}$ & $\begin{array}{l}\text { Gemcitabine + } \\
\text { Dacarbazine, } \\
\text { n (\%) }\end{array}$ & $\begin{array}{l}\text { Gemcitabine, } \\
\text { n (\%) }\end{array}$ & $\begin{array}{l}\text { Gemcitabine + } \\
\text { Docetaxel, } \\
\text { n (\%) }\end{array}$ & $\begin{array}{l}\text { Placebo, } \\
\text { n (\%) }\end{array}$ & $\begin{array}{l}\text { Sorafenib, } \\
\text { n (\%) }\end{array}$ & $\begin{array}{l}\text { Ifosfamide } \\
5 \mathrm{~g} / \mathrm{m} 2 / \text { day, } \\
\mathrm{n}(\%)\end{array}$ & $\begin{array}{l}\text { Ifosfamide } \\
3 \mathrm{~g} / \mathrm{m} 2 / \text { day, } \\
\mathrm{n}(\%)\end{array}$ \\
\hline Evaluable $\mathbf{N}$ & 240 & 123 & 130 & 130 & 52 & 57 & - & - & 2 & 2 & 27 & 31 \\
\hline \multicolumn{13}{|l|}{ Gl disorders } \\
\hline Abdominal pain & $0(0.0)$ & $0(0.0)$ & $6(4.6)$ & $6(4.6)$ & - & - & - & - & - & - & - & - \\
\hline Constipation & $1(0.4)$ & $3(2.4)$ & $0(0.0)$ & $2(1.5)$ & - & - & - & - & - & - & - & - \\
\hline Diarrhoea & $11(4.6)$ & $1(0.8)$ & $1(0.8)$ & $0(0.0)$ & $0(0.0)$ & $0(0.0)$ & - & - & - & - & - & - \\
\hline Gl pain & $6(2.5)$ & $5(4.1)$ & - & - & - & - & - & - & - & - & - & - \\
\hline $\begin{array}{l}\text { Mucositis/ } \\
\text { stomatitis }\end{array}$ & $1(0.4)$ & $0(0.0)$ & - & - & $0(0.0)$ & $1(1.8)$ & - & - & - & - & - & - \\
\hline Nausea & $8(3.3)$ & $2(1.6)$ & $7(5.4)$ & $3(2.3)$ & $1(1.9) \sim$ & $0(0.0) \sim$ & - & - & - & - & - & - \\
\hline Nausea/vomiting & - & - & - & - & - & - & - & - & - & - & $0(0.0) \$$ & - \\
\hline Vomiting & $8(3.3)$ & $1(0.8)$ & $7(5.4)$ & $2(1.5)$ & $0(0.0) \sim$ & $1(1.8) \sim$ & - & - & - & - & - & - \\
\hline $\begin{array}{l}\text { Small intestinal } \\
\text { obstruction }\end{array}$ & $3(1.3)$ & $0(0.0)$ & - & - & - & - & - & - & - & - & - & - \\
\hline \multicolumn{13}{|l|}{ General disorders } \\
\hline Asthenia & $0(0.0)$ & $0(0.0)$ & - & - & $5(9.6) \sim$ & $4(7.0) \sim$ & - & - & - & - & 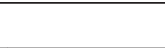 & - \\
\hline Back pain & - & - & $4(3.1)$ & $4(3.1)$ & - & - & - & - & - & - & - & - \\
\hline Fatigue & $33(13.7)$ & $6(4.9)$ & $10(7.7)$ & $9(6.9)$ & - & - & - & - & - & - & - & - \\
\hline Peripheral oedema & $5(2.1)$ & $2(1.6)$ & - & - & - & - & - & - & - & - & - & - \\
\hline Chest pain & $4(1.7)$ & $0(0.0)$ & & & & & & & & & & \\
\hline \multicolumn{13}{|c|}{ Skin and subcutaneous tissues disorders } \\
\hline Skin disorder & $4(1.7)$ & $0(0.0)$ & - & - & - & - & - & - & - & - & - & - \\
\hline \multicolumn{13}{|l|}{ Investigations } \\
\hline ALT increased & $23(9.6)$ & $4(3.3)$ & $62(47.7)$ & $12(9.2)$ & - & - & - & - & $0(0.0)$ & $0(0.0)$ & - & - \\
\hline $\begin{array}{l}\text { Alkaline } \\
\text { phosphatase }\end{array}$ & $7(2.9)$ & $1(0.8)$ & $2(1.5)$ & $3(2.3)$ & - & - & - & - & - & - & - & - \\
\hline AST increased & $19(7.9)$ & $2(1.6)$ & $41(31.5)$ & $4(3.1)$ & - & - & - & - & $0(0.0)$ & $0(0.0)$ & - & - \\
\hline $\begin{array}{l}\text { Creatinine } \\
\text { increased }\end{array}$ & $1(0.4)$ & $0(0.0)$ & $3(2.3)$ & $1(0.8)$ & - & - & - & - & - & - & - & - \\
\hline $\begin{array}{l}\text { Creatinine } \\
\text { phosphokinase }\end{array}$ & - & - & $7(5.4)$ & $12(9.2)$ & - & - & - & - & - & - & - & - \\
\hline Bilirubin increased & $3(1.3)$ & $2(1.6)$ & $1(0.8)$ & $1(0.8)$ & - & - & - & - & $0(0.0)$ & $0(0.0)$ & - & - \\
\hline $\begin{array}{l}\text { Gamma- } \\
\text { glutamyltransferase }\end{array}$ & $4(1.7)$ & $0(0.0)$ & - & - & - & - & - & - & - & - & - & - \\
\hline Weight loss & $9(3.8)$ & $0(0.0)$ & - & - & - & - & - & - & $0(0.0)$ & $0(0.0)$ & - & - \\
\hline
\end{tabular}


Table 3 Summary of grade 3 and/or 4 specific adverse events reported in $>1 \%$ patients across randomised controlled trials (Continued)

\begin{tabular}{|c|c|c|c|c|c|c|c|c|c|c|c|c|}
\hline $\begin{array}{l}\text { ENT examination } \\
\text { abnormal }\end{array}$ & $4(1.7)$ & $0(0.0)$ & - & - & - & - & - & - & - & - & - & - \\
\hline Hypoalbuminemia & - & - & - & - & - & - & - & - & $0(0.0)$ & $0(0.0)$ & - & - \\
\hline \multicolumn{13}{|l|}{ Hemorrhagic events } \\
\hline $\begin{array}{l}\text { Hemorrhagic } \\
\text { event (any) }\end{array}$ & $5(2.0)$ & $2(1.6)$ & - & - & - & - & - & - & $0(0.0)$ & $0(0.0)$ & - & - \\
\hline \multicolumn{13}{|c|}{ Metabolism and nutrition disorders } \\
\hline $\begin{array}{l}\text { Decreased } \\
\text { appetite }\end{array}$ & $14(5.9)$ & $0(0.0)$ & $1(0.8)$ & $0(0.0)$ & - & - & - & - & - & - & - & - \\
\hline Dehydration & $3(1.4)$ & $0(0.0)$ & - & - & - & - & - & - & - & - & - & - \\
\hline \multicolumn{13}{|c|}{ Musculoskeletal and connective tissue disorders } \\
\hline Arthralgia/myalgia & $5(2.1)$ & $0(0.0)$ & - & - & - & - & - & - & $0(0.0)$ & $0(0.0)$ & - & - \\
\hline $\begin{array}{l}\text { Musculoskeletal } \\
\text { pain }\end{array}$ & $5(2.1)$ & $2(1.6)$ & - & - & - & - & - & - & - & - & - & - \\
\hline \multicolumn{13}{|c|}{ Respiratory, thoracic, and medistinal disorders } \\
\hline Dyspnoea & $15(6.3)$ & $7(5.7)$ & $5(3.8)$ & $8(6.2)$ & - & - & - & - & - & - & - & - \\
\hline Pleural effusion & $5(2.1)$ & $0(0.0)$ & - & - & - & - & - & - & - & - & - & - \\
\hline \multicolumn{13}{|c|}{ Blood and lymphatic system disorders } \\
\hline Anaemia & $15(6.3)$ & $2(1.6)$ & $10(7.7)$ & $12(9.2)$ & $6(11.5)$ & $2(3.5)$ & - & - & - & - & - & - \\
\hline $\begin{array}{l}\text { Febrile } \\
\text { neutropenia }\end{array}$ & - & - & - & - & $3(5.8)$ & $5(8.8)$ & - & - & - & - & - & - \\
\hline Leukopenia & $3(1.3)$ & $0(0.0)$ & - & - & $16(30.8)$ & $15(26.3)$ & - & - & - & - & - & - \\
\hline Neutropenia & $10(4.2)$ & $0(0.0)$ & $61(46.9)$ & $17(13.1)$ & $17(32.7)$ & $27(47.4)$ & - & - & - & - & - & - \\
\hline Thrombocytopenia & $9(3.8)$ & $0(0.0)$ & $15(11.5)$ & $7(5.4)$ & $14(26.9)$ & $3(5.3)$ & - & - & - & - & $0(0.0) \$$ & - \\
\hline Lymphopenia & $23(9.6)$ & $13(10.6)$ & - & - & - & - & - & - & - & - & - & - \\
\hline \multicolumn{13}{|l|}{ Cardiac disease } \\
\hline Hypertension & $16(6.7)$ & $0(0.0)$ & - & - & - & - & - & - & - & - & - & - \\
\hline $\begin{array}{l}\text { Myocardial/LVEF } \\
\text { Dysfunction }\end{array}$ & $4(1.7)$ & $0(0.0)$ & - & - & - & - & - & - & - & - & - & - \\
\hline \multicolumn{13}{|l|}{ Other disorders } \\
\hline Tumour pain & $20(8.3)$ & $9(7.3)$ & & & & & & & & & & \\
\hline $\begin{array}{l}\text { Non- } \\
\text { haemotological }\end{array}$ & - & - & - & - & - & - & - & 29 & - & - & - & - \\
\hline
\end{tabular}


Table 4 Results of treatment discontinuations across randomised controlled trials

\begin{tabular}{|c|c|c|c|c|c|c|c|c|c|c|}
\hline \multirow[t]{2}{*}{ Intervention } & \multirow[t]{2}{*}{ Study } & \multirow[t]{2}{*}{$\mathbf{N}$} & \multicolumn{7}{|c|}{ Treatment discontinuation, $\mathrm{n}(\%)$} & \multirow[t]{2}{*}{ Most common AEs leading to discontinuations } \\
\hline & & & All & Due to $A E$ & $\begin{array}{l}\text { Due to } \\
\text { death }\end{array}$ & Due to PD & $\begin{array}{l}\text { Due to lost } \\
\text { to follow-up }\end{array}$ & $\begin{array}{l}\text { Due to patient } \\
\text { decision }\end{array}$ & $\begin{array}{l}\text { Due to other } \\
\text { reasons }\end{array}$ & \\
\hline Pazopanib & $\begin{array}{l}\text { PALETTE study } \\
2011 / 2\end{array}$ & 246 & $240(97.6)$ & $41(16.7)$ & $3(1.2)$ & $178(72.4)$ & $0(0.0)$ & $14(5.7)$ & $4(1.6) 9$ & $\begin{array}{l}\text { ALT elevation, dyspnoea, left ventricular dysfunction, } \\
\text { fatigue, hypertension, vomiting, depressed mood, } \\
\text { embolism, nausea, pericardial effusion, and small } \\
\text { intestinal obstruction }\end{array}$ \\
\hline Placebo & $\begin{array}{l}\text { PALETTE study } \\
2011 / 2\end{array}$ & 123 & $123(100)$ & $3(2.4)$ & $0(0)$ & $119(96.7)$ & $0(0.0)$ & $1(0.8)$ & $0(0.0)$ & Dyspnoea \\
\hline Trabectedin 1.5 mg/m2 q3w & Demetri 2009 & 136 & $128(94.1)$ & $12(8.8)$ & $2(1.5)$ & $89(65.4)$ & $2(1.5)$ & $17(12.5)$ & $6(4.4) \dagger$ & AEs leading to disconsolation were not reported \\
\hline Trabectedin 0.58 mg/m2 qw & Demetri 2009 & 134 & $134(100)$ & $10(7.5)$ & $3(2.2)$ & $94(70.1)$ & $0(0.0)$ & $6(4.5)$ & $21(15.7) \neq$ & AEs leading to disconsolation were not reported \\
\hline Dacarbazine & GEIS study 2011 & 54 & $50(92.6)$ & $2(3.7)$ & - & $41(75.9)$ & $0(0.0)$ & - & $7(13.0)$ & - \\
\hline Gemcitabine + Dacarbazine & GEIS study 2011 & 59 & $47(79.7)$ & $0(0.0)$ & - & $36(61.0)$ & $0(0.0)$ & $1(1.7)$ & $10(16.9)$ & - \\
\hline Gemcitabine & Pautier 2009 & $84^{*}$ & - & 3 & - & - & - & - & - & AEs leading to disconsolation were not reported \\
\hline Gemcitabine + Docetaxel & Pautier 2009 & & - & 9 & - & - & - & - & - & Hypersensitivity in one patient \\
\hline
\end{tabular}

AE: Adverse Events; N: Number of Patients; n: Number with Outcome; PD: Progressive Disease; q3w: Every Three Weeks; qw: Every Week; -Represents data not reported; *Represents total population randomised in the study (patients randomised in each arm not reported); IIncludes three patients discontinuing treatment due to protocol violation; +Includes patients discontinuing due to screening failure $(\mathrm{n}=3$ ) and ineligibility of patients $(n=2)$; $\neq$ Includes patients discontinuing due to screening failure $(n=2)$, cross-over before progression $(n=14)$, and ineligibility of patients $(n=1)$. 
Table 5 Summary of relevant prospective non-randomised trials (with sample size more than 10) included in the review

\begin{tabular}{|c|c|c|c|c|}
\hline Intervention & Dose & Study & $\mathbf{N}$ & STS subtypes (\%) \\
\hline Brostallicin & $10 \mathrm{mg} / \mathrm{m}^{2}$ & Leahy 2007 [33] & 43 & Multiple \\
\hline Cisplatin & $50 \mathrm{mg} / \mathrm{m}^{2}$ & Thigpen 1986 [34] & 20 & Leiomyosarcoma \\
\hline Cyclophosphamide & $1.5 \mathrm{~g} / \mathrm{m}^{2}$ & Bramwell $1993[35,36] \sim$ & 18 & NS \\
\hline Dacarbazine & $1200 \mathrm{mg} / \mathrm{m}^{2}$ & Buesa 1991 [73] & 47 & Multiple \\
\hline Docetaxel & $100 \mathrm{mg} / \mathrm{m}^{2}$ & Kostler 2001 [37] & 25 & Multiple \\
\hline Docetaxel & $100 \mathrm{mg} / \mathrm{m}^{2}$ & Santoro 1999 [38] & 37 & Multiple \\
\hline Docetaxel & $100 \mathrm{mg} / \mathrm{m}^{2}$ & van Hoesel $1994[39,40] \sim$ & 21 & NS \\
\hline Doxorubicin & $75 \mathrm{mg} / \mathrm{m}^{2}$ & Mouridsen 1987 [41] & 23 & NS \\
\hline Etoposide & $200-240 \mathrm{mg} / \mathrm{m}^{2} /$ day & Crawley 1997 [42] & 17 & Multiple \\
\hline Etoposide & $130 \mathrm{mg} / \mathrm{m}^{2}$ & Dombernowsky 1987 [43] & 26 & NS \\
\hline Gefitinib & $500 \mathrm{mg}$ & Ray-Coquard 2008 [44] & 48 & Synovial sarcoma $\approx$ \\
\hline Gemcitabine & $1000 \mathrm{mg} / \mathrm{m}^{2}$ & Ferraresi 2008 [74] & 14 & Multiple \\
\hline Gemcitabine & $1000 \mathrm{mg} / \mathrm{m}^{2}$ & Hartmann 2006 [45] & 15 & Multiple \\
\hline Gemcitabine & $1000 \mathrm{mg} / \mathrm{m}^{2}$ & Look 2004 [75] & 35 & Leiomyosarcoma \\
\hline Gemcitabine & $200-250 \mathrm{mg} / \mathrm{m}^{2}$ & Spath-Schwalbe 2000 [46] & 18 & Multiple \\
\hline Ifosfamide & $2 \mathrm{~g} / \mathrm{m}^{2}$ for $4 \mathrm{~d} \mathrm{q} 3 \mathrm{w}$ & Antman $1985[47]$ & 31 & Multiple \\
\hline Ifosfamide & $2 \mathrm{~g} / \mathrm{m}^{2}$ for $4 \mathrm{~d} \mathrm{q} 3 \mathrm{w}$ & Antman 1989 [48] & 94 & Multiple \\
\hline Ifosfamide & $1 \mathrm{~g} / \mathrm{m}^{2}$ daily until Gr3 granulocytopenia q4w & Babovic 1998 [76] & 21 & Multiple \\
\hline Ifosfamide & $4 \mathrm{~g} / \mathrm{m}^{2}$ for $3 \mathrm{~d} \mathrm{q} 4 \mathrm{w}$ & Le Cesne $1995[49,77] \sim$ & 40 & Multiple \\
\hline Ifosfamide & $4 \mathrm{~g} / \mathrm{m}^{2}$ for $3 \mathrm{~d} \mathrm{q} 4 \mathrm{w}$ & Nielsen 2000 [78] & 13 & NS \\
\hline Ifosfamide & $3.5 \mathrm{~g} / \mathrm{m}^{2}$ for $4 \mathrm{~d} \mathrm{q} 3 \mathrm{w}$ & Palumbo 1997 [79] & 38 & Multiple \\
\hline Ifosfamide & $4 \mathrm{~g} / \mathrm{m}^{2}$ for $3.5 \mathrm{~d} \mathrm{q} 3 \mathrm{w}$ & Patel 1997 [80] & 12 & Multiple \\
\hline Ifosfamide & $2 \mathrm{~g} / \mathrm{m}^{2}$ loading dose followed by $4 \mathrm{~g} / \mathrm{m}^{2}$ for $3 \mathrm{~d} \mathrm{q} 3 \mathrm{w}$ & Patel 1997 [80] & 32 & Multiple \\
\hline Ifosfamide & $60 \mathrm{mg} / \mathrm{kg}$ for $5 \mathrm{~d}$ q3-4w & Scheulen 1983 [81] & 16 & NS \\
\hline Liposomal doxorubicin & $55 \mathrm{mg} / \mathrm{m}^{2}$ & Skubitz 2003 [50] & 20 & Multiple \\
\hline Liposomal doxorubicin & $30-50 \mathrm{mg} / \mathrm{m}^{2}$ & Toma 2000 [51] & 25 & Multiple \\
\hline Methotrexate & $40 \mathrm{mg} / \mathrm{m}^{2}$ & Buesa 1984 [52] & 37 & NS \\
\hline Paclitaxel & $135-175 \mathrm{mg} / \mathrm{m}^{2}$ & Palumbo 1997 [53] & 12 & Multiple \\
\hline Paclitaxel & $200 \mathrm{mg} / \mathrm{m}^{2}$ & Patel 1997 [54] & 12 & Multiple \\
\hline Paclitaxel & $120 \mathrm{mg} / \mathrm{m}^{2}$ & Skubitz 1997 [55] & 17 & Multiple \\
\hline Sorafenib & $400 \mathrm{mg}$ & Bertuzzi $2010[56,57] \sim$ & 61 & Multiple \\
\hline Sorafenib & $400 \mathrm{mg}$ & Pacey 2011 [28] & 16 & Multiple \\
\hline Sunitinib & $50 \mathrm{mg}$ & Decoster 2010 [58] & 24 & NS \\
\hline Trabectedin & $1.5 \mathrm{mg} / \mathrm{m}^{2}$ & $\begin{array}{l}\text { Garcia-Carbonero } 2004 \\
{[59,60,82] \sim}\end{array}$ & 36 & Multiple \\
\hline Trabectedin & $1.5 \mathrm{mg} / \mathrm{m}^{2}$ & Le Cesne 2005 [83] & 104 & Multiple \\
\hline Trabectedin & $1.5 \mathrm{mg} / \mathrm{m}^{2}$ & Yovine $2004[61,84] \sim$ & 27 & Multiple \\
\hline Biricodar + doxorubicin & B - $120 \mathrm{mg} 1.5 \mathrm{mg} / \mathrm{m}^{2} / \mathrm{hr} ; \mathrm{D}-60 \mathrm{mg} / \mathrm{m}^{2}$ & Bramwell 2002 [62] & 18 & Multiple \\
\hline Cisplatin + ifosfamide & $\mathrm{C}-100 \mathrm{mg} / \mathrm{m}^{2}$ day $2 \& 9 ; 1-2.5 \mathrm{~g} / \mathrm{m}^{2}$ for $3 \mathrm{~d}$ & Budd 1993 [85] & 38 & Multiple \\
\hline Cisplatin + vinblastine & $C-50-100 \mathrm{mg} / \mathrm{m}^{2}$ day $1 ; \mathrm{V}-1.0-1.2 \mathrm{mg} / \mathrm{m}^{2}$ for $5 \mathrm{~d}$ & Keohan 1997 [63] & 18 & Multiple \\
\hline $\mathrm{D}+\mathrm{IL}-2$ & $\mathrm{D}-70 \mathrm{mg} / \mathrm{m}^{2} ; \mathrm{IL}-2-18 \mathrm{MIU} / \mathrm{m}^{2}$ & Le Cesne 1999 [64] & 12 & NS \\
\hline Epirubicin + lonidamine & $\mathrm{E}-120 \mathrm{mg} / \mathrm{m}^{2} ; \mathrm{l}-150-450 \mathrm{mg}$ & Lopez 1995 [65] & 25 & Multiple \\
\hline Etoposide + ifosfamide & $E-200 \mathrm{mg} / \mathrm{m}^{2}$ for $3 \mathrm{~d} ; \mathrm{l}-1.5 \mathrm{~g} / \mathrm{m}^{2}$ for $3 \mathrm{~d}$ & Saeter 1995 [86] & 11 & NS \\
\hline Etoposide + ifosfamide & $E-50 \mathrm{mg} / \mathrm{m}^{2}$ for $8 \mathrm{~d} ; \mathrm{l}-1.5 \mathrm{~g} / \mathrm{m}^{2}$ for $6 \mathrm{~d}$ & Skubitz 1993 [87] & 16 & Multiple \\
\hline Gemcitabine + dacarbazine & $\mathrm{G}-800-2160 \mathrm{mg} / \mathrm{m}^{2} ; \mathrm{DTIC}-500 \mathrm{mg} / \mathrm{m}^{2}$ & Buesa 2004 [66] & 22 & Multiple \\
\hline Gemcitabine + dacarbazine & $\mathrm{G}-1800 \mathrm{mg} / \mathrm{m}^{2} ; \mathrm{DTIC}-500 \mathrm{mg} / \mathrm{m}^{2}$ & Losa 2007 [67] & 26 & Multiple \\
\hline Gemcitabine + docetaxel & $\mathrm{G}-900 \mathrm{mg} / \mathrm{m}^{2} ; \mathrm{D}-100 \mathrm{mg} / \mathrm{m}^{2}$ & Hensley $2002[88]$ & 16 & Leiomyosarcoma \\
\hline
\end{tabular}


Table 5 Summary of relevant prospective non-randomised trials (with sample size more than 10) included in the review (Continued)

\begin{tabular}{lllll}
\hline Gemcitabine + docetaxel & $\mathrm{G}-900 \mathrm{mg} / \mathrm{m}^{2} ; \mathrm{D}-100 \mathrm{mg} / \mathrm{m}^{2}$ & Hensley 2008 [89] & 51 & Leiomyosarcoma \\
\hline Gemcitabine + docetaxel & $\mathrm{G}-900 \mathrm{mg} / \mathrm{m}^{2} ; \mathrm{D}-100 \mathrm{mg} / \mathrm{m}^{2}$ & Montalar 2008 [68] & 12 & Multiple \\
\hline Methotrexate + vincristine & $\mathrm{M}-5 \mathrm{~g} / \mathrm{m}^{2} ; \mathrm{V}-1 \mathrm{mg} / \mathrm{m}^{2}$ & Vaughn 1984 [69] & 14 & $\mathrm{NS}$ \\
\hline VAC + IE & $\mathrm{V}-2 \mathrm{mg} ; \mathrm{A}-70 \mathrm{mg} / \mathrm{m}^{2} ; \mathrm{C}-600 \mathrm{mg} / \mathrm{m}^{2} ;$ & Palumbo 1998 [70] & 12 & $\mathrm{NS}$ \\
\hline $\mathrm{I}+\mathrm{VC}+\mathrm{D}+\mathrm{DTIC}+\mathrm{IL}-2$ & $\mathrm{IL}-2-18 \mathrm{~m}$ units/d, followed by CYVADIC & Gravis 2001 [71] & 1 & Leiomyosarcoma \\
\hline $\mathrm{D}+\mathrm{I}+\mathrm{DTIC}+\mathrm{IL}-2$ & $\mathrm{IL}-2-18 \mathrm{~m}$ units/d, followed by D+I+DTIC & Gravis 2001 [71] & 9 & Multiple \\
\hline D + IL-2 & $\mathrm{IL}-2-18 \mathrm{~m}$ units $/ \mathrm{d}$, followed by D & Gravis 2001 [71] & 3 & Multiple \\
\hline Carboplatin + etoposide & $\mathrm{C}-300 \mathrm{mg} / \mathrm{m}^{2} ; \mathrm{E}-300 \mathrm{mg} / \mathrm{m}^{2}$ & Holstein 1996 [72] & 8 & Multiple \\
\hline Dacarbazine & $1200 \mathrm{mg} / \mathrm{m}^{2}$ & Holstein 1996 [72] & 14 & Multiple \\
\hline
\end{tabular}

C + VC + D + DTIC + IL-2: Cyclophosphamide + Vincristine + Doxorubicin + Dacarbazine + Interleukin-2; D + IL-2: Doxorubicin + Interleukin-2; D + I + DTIC + IL-2: Doxorubicin + Ifosfamide + Dacarbazine + Interleukin-2; N: Number of Included Patients; n-RCT: Non-Randomised Controlled Trials; VAC + IE: Vincristine + Adriamycin + Cyclophosphamide + Ifosfamide + Etoposide; Represents secondary reference; NS: Not Specified; $\approx$ Comprise $4.2 \%$ other soft tissue sarcoma.

assessing ifosfamide monotherapy, variable activity was observed in terms of response rate $(4.8 \%$ [76] to $62.5 \%$ [81]) (Table 6). Although a wide variety of doses and schedules was used in these trials, with cumulative dose per cycle ranging from 8 to $14 \mathrm{mg} / \mathrm{m}^{2}$, this did not have a clear impact on efficacy. Gemcitabine as monotherapy (four studies) and in combination therapy demonstrated a variable efficacy in terms of median OS (monotherapy: 6.0 months to 11.8 months; combination therapy: 14.7 months) and ORR (monotherapy: $6.7 \%$ to $21.2 \%$; combination therapy: $3.8 \%$ to $50.0 \%)$. However, a superior response rate $(>20 \%)$ was observed with gemcitabine monotherapy ( $21.2 \%$ vs. $6.7 \%$ to $11.1 \%$ ) and gemcitabine plus docetaxel (25.5\% to $50.0 \%$ vs. $8.3 \%$ ) among patients with uterine leiomyosarcoma compared with patients with mixed STS subtypes (Table 6). Once more, there were no clear trends relating to dose of gemcitabine.

The only treatment that demonstrated a similar antitumour activity across different trials was trabectedin (three studies). Trabectedin was associated with a response rate ranging from $7.4 \%$ [84] to $8.5 \%$ [83] and median OS varying between 9.1 months [77] and 12.1 months [82]. Limited anti-tumour activity (ORR $\leq 5 \%)$ in pre-treated patients with STS was demonstrated by treatments including brostallicin, cisplatin, cyclophosphamide, dacarbazine, gefitinib, methotrexate, and sunitinib [90]. Summaries of the efficacy results for interventions evaluated across non-randomised studies are presented in Table 6.

\section{Safety results}

Across the non-randomised studies identified in the review, haematological AEs were the most commonly reported AEs, particularly with therapies including docetaxel, gemcitabine, docetaxel plus gemcitabine, etoposide, carboplatin plus etoposide, cisplatin plus ifosfamide, and trabectedin. However, AEs were not reported in a sufficiently consistent manner for a meaningful comparison across studies. The details of the grade 3 and/or 4 AEs observed across these studies are provided in an additional file (see Additional file 7).

Data related to treatment discontinuations due to AEs were reported in only three of the included studies. Thus, it was difficult to draw any conclusions regarding the comparative tolerability of the evaluated interventions $[74,82,83]$.

\section{Discussion}

The objective of this systematic review was to comprehensively review available evidence on the efficacy and safety of treatments used for advanced STS following prior therapy with anthracycline- and/or ifosfamide for advanced disease. Due to the paucity of RCT evidence in this anthracyline pre-treated setting, this review included RCTs and non-randomised studies (prospective and retrospective) to allow for a detailed description of the evidence supporting these interventions being used for the management of patients with pre-treated advanced STS.

The available RCT evidence (from six studies) suggests that pazopanib, trabectedin, and the combination of gemcitabine and dacarbazine are effective treatments for pre-treated patients with advanced STS. These agents were also among those identified as potentially active second-line treatments in a recent analysis of Phase II studies by Penel and colleagues [91]. Pazopanib has demonstrated a significant advantage over placebo with an increase of 3 months in median PFS $[18,19]$. Treatment with q3w 24-hour dosing schedule of trabectedin was associated with significantly greater median PFS and TTP compared with the qw 3-hour schedule [22-26], and the combination of gemcitabine and dacarbazine was more effective than dacarbazine monotherapy in terms of 3-month PFS rate, median PFS, and median OS [20,21]. These findings should be interpreted in view of the fact that the evidence comes from Phase II studies except for the pazopanib PALETTE study. The primary aim of 
Table 6 Efficacy/Activity outcomes across prospective non-randomised trials with sample size more than 10

\begin{tabular}{|c|c|c|c|c|c|c|c|c|c|c|c|c|}
\hline \multirow[t]{2}{*}{ Intervention } & \multirow[t]{2}{*}{ Study } & \multirow[t]{2}{*}{$\mathrm{N}$} & \multicolumn{5}{|c|}{ Response rate $\mathrm{n}(\%), \mathrm{IRC}$ or unclear } & \multirow{2}{*}{$\begin{array}{l}\text { PFS } \\
n(\%) \text { or median } \\
(95 \% \mathrm{Cl})\end{array}$} & \multirow{2}{*}{$\begin{array}{l}\text { TTP in } \\
\text { months } \\
\text { Median } \\
\text { (range) }\end{array}$} & \multirow{2}{*}{$\begin{array}{l}\text { OS } \\
\text { n (\%) or median } \\
\text { (range) }\end{array}$} & \multirow{2}{*}{$\begin{array}{l}\text { DOR in } \\
\text { months } \\
\text { Median } \\
\text { (range) }\end{array}$} & \multirow{2}{*}{$\begin{array}{l}\text { Progressive } \\
\text { disease } \\
\mathrm{n}(\%), \text { IRC or } \\
\text { unclear }\end{array}$} \\
\hline & & & ORR & CR & PR & MR & SD & & & & & \\
\hline Brostallicin & Leahy 2007 & 40 & $2(5.0 \%) \sim$ & $0(0.0 \%) \sim$ & $2(5.0 \%) \sim$ & - & $\begin{array}{l}20(50.0 \%) \\
\sim\end{array}$ & $\begin{array}{l}3 \text { mo: } 18(45.0 \%) \sim ; \\
6 \text { mo: } 9(22.5 \%)^{\sim}\end{array}$ & $\begin{array}{l}2.9 \text { months } \\
(1.4-3.7)\end{array}$ & $\begin{array}{l}7.6 \text { months } \\
(5.2-13.8)\end{array}$ & - & $17(42.5 \%) \sim$ \\
\hline Cisplatin & Thigpen 1986 & 19 & $1(5.3 \%)$ & $1(5.3 \%)$ & $0(0.0 \%)$ & - & $7(36.8 \%)$ & - & - & - & $\begin{array}{l}9 \text { months } \\
(\mathrm{N}=1)\end{array}$ & $11(57.9 \%)$ \\
\hline Cyclophosphamide & Bramwell 1993 & 18 & $0(0.0 \%)$ & $0(0.0 \%)$ & $0(0.0 \%)$ & - & - & - & - & - & - & - \\
\hline Dacarbazine & Buesa 1991 & 44 & $8(18.2 \%)$ & $1(2.3 \%)$ & $7(15.9 \%)$ & - & $8(18.2 \%)$ & - & - & - & - & $28(63.6 \%)$ \\
\hline Docetaxel & Kostler 2001 & 25 & $4(16.0 \%)$ & $0(0.0 \%)$ & $4(16.0 \%)$ & - & - & - & - & - & - & - \\
\hline Docetaxel & Santoro 1999 & 36 & 1 (2.8\%)\# & $0(0.0 \%)$ & $1(2.8 \%)$ & - & $10(27.8 \%)$ & 1.4 months $(\mathrm{N}=37)$ & - & $\begin{array}{l}11.5 \text { months } \\
(\mathrm{N}=37)\end{array}$ & - & 25 (69.4\%) \\
\hline Docetaxel & Van Hoesel 1994 & 21 & - & $0(0.0 \%) \sim$ & - & - & - & - & - & - & - & - \\
\hline Doxorubicin & Mouridsen 1987 & 23 & $2(8.7 \%) \sim$ & - & - & - & - & - & - & - & - & - \\
\hline Etoposide & Crawley 1997 & 16 & $0(0.0 \%)$ & $0(0.0 \%)$ & $0(0.0 \%)$ & - & $8(50.0 \%)$ & - & - & $\begin{array}{l}3.7 \text { months } \\
(2.1-5.2)^{*} \\
(\mathrm{~N}=17)\end{array}$ & - & $8(50.0 \%)$ \\
\hline Etoposide & $\begin{array}{l}\text { Dombernowsky } \\
1987\end{array}$ & 26 & - & - & $1(3.8 \%)$ & - & - & - & - & - & $\begin{array}{l}19 \text { months } \\
(\mathrm{N}=1)\end{array}$ & - \\
\hline Gefitinib & $\begin{array}{l}\text { Ray-Coquard } \\
2008\end{array}$ & 46 & $0(0.0 \%) \sim$ & $0(0.0 \%) \sim$ & $0(0.0 \%) \sim$ & $0(0.0 \%) \sim$ & $\begin{array}{l}10(21.7 \%) \\
\sim\end{array}$ & $\begin{array}{l}4 \mathrm{mo:} 10(21.7 \%)^{\sim} ; \\
6 \mathrm{mo:} 3(6.5 \%)^{\sim}\end{array}$ & 1.4 months & - & - & $32(69.6 \%) \sim$ \\
\hline Gemcitabine & Ferraresi 2008 & 14 & $1(7.1 \%)$ & $0(0.0 \%)$ & $1(7.1 \%)$ & - & $3(21.4 \%)$ & - & $\begin{array}{l}3.1 \text { months } \\
(1.0-9.5)\end{array}$ & $\begin{array}{l}11.8 \text { months } \\
(1.0-54.5)\end{array}$ & $\begin{array}{l}6.5 \text { months } \\
(\mathrm{N}=1)\end{array}$ & $10(71.4 \%)$ \\
\hline Gemcitabine & Hartmann 2006 & 15 & $1(6.7 \%)$ & $0(0.0 \%)$ & $1(6.7 \%)$ & - & $7(46.7 \%)$ & $\begin{array}{l}3 \text { mo: } 7(46.7 \%) ; \\
6 \text { mo: } 2(13.3 \%) ; \\
3.0 \text { months }(1.0-33.0)\end{array}$ & 3 months & $\begin{array}{l}6.0 \text { months } \\
(3.0-33.0)\end{array}$ & - & 7 (46.7\%) \\
\hline Gemcitabine & Look 2004 & 33 & 7 (21.2\%) & $1(3.0 \%)$ & $6(18.2 \%)$ & - & - & - & - & - & - & - \\
\hline Gemcitabine & $\begin{array}{l}\text { Spath-Schwalbe } \\
2000\end{array}$ & 18 & 2 (11.1\%)\# & $0(0.0 \%)$ & $2(11.1 \%)$ & - & $6(33.3 \%)$ & - & - & $\begin{array}{l}12 \text { mo: } 5 \\
(27.8 \%) ; 8 \text { months }\end{array}$ & $\begin{array}{l}5.5 \text { months } \\
(5.0-6.0) \\
(N=2)\end{array}$ & $9(50.0 \%)$ \\
\hline Ifosfamide & Antman 1985 & 26 & 8 (30.8\%)\# & $0(0.0 \%)$ & $8(30.8 \%)$ & $3(11.5 \%)$ & $11(42.3 \%)$ & - & - & - & $\begin{array}{l}(2.0-10.0+) \\
(N=11)\end{array}$ & $4(15.4 \%)$ \\
\hline Ifosfamide & Antman 1989 & 94 & $17(18.1 \%)$ & $2(2.1 \%)$ & - & - & - & - & - & - & - & - \\
\hline Ifosfamide & Babovic 1998 & 21 & $1(4.8 \%)$ & $1(4.8 \%)$ & $0(0.0 \%)$ & - & $4(19.0 \%)$ & - & - & - & - & 16 (76.2\%) \\
\hline Ifosfamide & Le Cesne 1995 & 36 & 12 (33.3\%) & $0(0.0 \%)$ & $12(33.3 \%)$ & - & $8(22.2 \%)$ & - & - & $\begin{array}{l}20.0 \text { months } \\
(6.0-91.0+) \\
(\mathrm{N}=40)\end{array}$ & $\begin{array}{l}8.0 \text { months } \\
(6.0-13.0+) \\
(\mathrm{N}=12)\end{array}$ & $16(44.4 \%)$ \\
\hline
\end{tabular}


Table 6 Efficacy/Activity outcomes across prospective non-randomised trials with sample size more than 10 (Continued)

\begin{tabular}{|c|c|c|c|c|c|c|c|c|c|c|c|c|}
\hline Ifosfamide & Palumbo 1997a & 38 & 15 (39.5\%) & $1(2.6 \%)$ & $14(36.8 \%)$ & - & $17(44.7 \%)$ & - & - & $\begin{array}{l}13.0 \text { months } \\
(6.0-30.0+)\end{array}$ & $\begin{array}{l}9.0 \text { months } \\
(5.0-21.0+) \\
(\mathrm{N}=15)\end{array}$ & $6(15.8)$ \\
\hline Ifosfamide & Patel 1997b & 11 & $5(45.5 \%)$ & $0(0.0 \%)$ & $5(45.5 \%)$ & - & $2(18.2 \%)$ & - & - & - & - & $4(36.4 \%)$ \\
\hline Ifosfamide & Patel 1997b & 32 & $6(18.8 \%)$ & $2(6.3 \%)$ & $4(12.5 \%)$ & - & - & - & - & - & - & - \\
\hline Ifosfamide & Scheulen 1983 & 16 & $10(62.5 \%)$ & $2(12.5 \%)$ & $3(18.8 \%)$ & $5(31.3 \%)$ & $1(6.3 \%)$ & - & - & - & - & $5(31.3 \%)$ \\
\hline $\begin{array}{l}\text { Liposomal } \\
\text { doxorubicin }\end{array}$ & Skubitz 2003 & 20 & 1 (5.0\%)\# & $1(5.0 \%)$ & $0(0.0 \%)$ & $3(15.0 \%)$ & $3(15.0 \%)$ & - & - & - & - & $12(60.0 \%)$ \\
\hline $\begin{array}{l}\text { Liposomal } \\
\text { doxorubicin }\end{array}$ & Toma 2000 & 25 & $3(12.0 \%)$ & $0(0.0 \%)$ & $3(12.0 \%)$ & $2(8.0 \%)$ & $17(68.0 \%)$ & - & - & $\begin{array}{l}12.0 \text { months } \\
(6.0-16.0+)\end{array}$ & $\begin{array}{l}(3.0-9.0+) \\
(\mathrm{N}=3)\end{array}$ & $3(12.0 \%)$ \\
\hline Methotrexate & Buesa 1984 & 21 & $0(0.0 \%)$ & $0(0.0 \%)$ & $0(0.0 \%)$ & - & - & - & - & - & - & - \\
\hline Paclitaxel & Palumbo 1997b & 12 & 1 (8.3\%)\# & $0(0.0 \%)$ & $1(8.3 \%)$ & $1(8.3 \%)$ & $6(50.0 \%)$ & - & 3 months & 6 months & $\begin{array}{l}4 \text { months ( } N \\
=1 \text { ) }\end{array}$ & 4 (33.3\%) \\
\hline Paclitaxel & Patel 1997a & 12 & $0(0.0 \%)$ & $0(0.0 \%)$ & $0(0.0 \%)$ & - & - & - & - & - & & - \\
\hline Paclitaxel & Skubitz 1997 & 15 & 1 (6.7\%)\# & 0 & $1(6.7 \%)$ & - & - & - & - & - & $\begin{array}{l}12 \text { months ( } N \\
=1 \text { ) }\end{array}$ & - \\
\hline Sorafenib & Bertuzzi 2010 & 61 & - & - & $9(14.8 \%)$ & - & - & 6 mo: 20 (32.7\%) & - & $\begin{array}{l}6 \mathrm{mo}: 41 \\
(67.2 \%)(\mathrm{N}=61)\end{array}$ & - & - \\
\hline Sorafenib & Bertuzzi 2010 & $30 \$$ & 2 (6.7\%)\# & $1(3.3 \%)$ & $1(3.3 \%)$ & - & $18(60.0 \%)$ & - & - & - & - & $10(33.3 \%)$ \\
\hline Sorafenib & Pacey 2011 & 16 & - & - & - & - & $0(0.0 \%)$ & - & - & - & - & - \\
\hline Sunitinib & Decoster 2010 & 20 & 1 (5.0\%)\# & $0(0.0 \%)$ & $1(5.0 \%)$ & - & $4(20.0 \%)$ & - & - & - & $\begin{array}{l}8.3 \text { months } \\
(\mathrm{N}=1)\end{array}$ & $15(75.0 \%)$ \\
\hline Trabectedin & $\begin{array}{l}\text { Garcia-Carbonero } \\
2004\end{array}$ & 36 & $3(8.3 \%)$ & $1(2.8 \%)$ & $2(5.6 \%)$ & $2(5.6 \%)$ & - & 12 mo: 4 (11.1\%) & $\begin{array}{l}1.7 \text { months } \\
(1.3-4.4)^{*}\end{array}$ & $\begin{array}{l}6 \text { mo: } 27(75.0 \%) ; \\
12 \text { mo: } 19(52.7 \%) ; \\
12.1 \text { months } \\
(8.1-26.5)^{*}\end{array}$ & $\begin{array}{l}9.0 \text { months } \\
(4.0-20.0) \\
(N=3)\end{array}$ & - \\
\hline Trabectedin & Le Cesne 2005 & 104 & $\begin{array}{l}8(8.7 \%) \sim \\
(N=92)\end{array}$ & $\begin{array}{l}0(0.0 \%) \sim \\
(\mathrm{N}=92)\end{array}$ & $\begin{array}{l}8(8.7 \%) \sim \\
(\mathrm{N}=92)\end{array}$ & - & $\begin{array}{l}44(47.8 \%) \\
\sim+(\mathrm{N}=92)\end{array}$ & $\begin{array}{l}3 \text { mo: } 54(52 \%) \sim ; \\
6 \text { mo: } 30(29 \%) \sim ; \\
9 \text { mo: } 21(20 \%) \sim ; \\
12 \text { mo: } 18(17 \%) \sim\end{array}$ & $\begin{array}{l}3.4 \text { months } \\
(2.5-4.1)^{*} \\
\sim(\mathrm{N}=99)\end{array}$ & $\begin{array}{l}12 \text { mo: } 44(42 \%) ; \\
9.1 \text { months } \\
(7.8-12.1)^{*}\end{array}$ & $\begin{array}{l}11.6 \text { months } \\
(\mathrm{N}=8)\end{array}$ & $\begin{array}{l}35(38.0 \%) \sim \\
(N=92)\end{array}$ \\
\hline Trabectedin & Yovine 2004 & 27 & $2(7.4 \%) \sim$ & $0(0.0 \%) \sim$ & $2(7.4 \%) \sim$ & $2(7.4 \%) \sim$ & $\begin{array}{l}4(14.8 \%) \\
\sim\end{array}$ & - & - & - & $\begin{array}{l}12.2 \text { months } \\
(\mathrm{N}=2)\end{array}$ & - \\
\hline $\begin{array}{l}\text { Biricodar + } \\
\text { doxorubicin }\end{array}$ & Bramwell 2002 & 15 & $\begin{array}{l}2(13.3 \%) \\
\sim \#\end{array}$ & $0(0.0 \%) \sim$ & $\begin{array}{l}2(13.3 \%) \\
\sim\end{array}$ & - & $\begin{array}{l}7(46.7 \%) \\
\sim\end{array}$ & 3.1 months $\sim(\mathrm{N}=15)$ & - & - & - & $6(40.0 \%) \sim$ \\
\hline $\begin{array}{l}\text { Cisplatin + } \\
\text { ifosfamide }\end{array}$ & Budd 1993 & 38 & $8(21.0 \%)$ & $3(7.9 \%)$ & $5(13.2 \%)$ & - & - & - & - & 11 months & - & - \\
\hline $\begin{array}{l}\text { Cisplatin + } \\
\text { vinblastine }\end{array}$ & Keohan 1997 & 15 & $0(0.0 \%)$ & $0(0.0 \%)$ & $0(0.0 \%)$ & - & 7 (46.7\%) & - & $\begin{array}{l}2.0 \text { months } \\
(0.7-12.0) \\
(\mathrm{N}=18)\end{array}$ & - & - & 8 (53.3\%) \\
\hline$D+I L-2$ & Le Cesne 1999 & 12 & $2(16.7 \%)$ & $0(0.0 \%)$ & $2(16.7 \%)$ & - & - & - & - & - & - & - \\
\hline
\end{tabular}


Table 6 Efficacy/Activity outcomes across prospective non-randomised trials with sample size more than 10 (Continued)

\begin{tabular}{|c|c|c|c|c|c|c|c|c|c|c|c|c|}
\hline $\begin{array}{l}\text { Epirubicin + } \\
\text { lonidamine }\end{array}$ & Lopez 1995 & 24 & $2(8.3 \%)$ & $0(0.0 \%)$ & $2(8.3 \%)$ & - & $1(4.2 \%)$ & - & - & $\begin{array}{l}14 \text { months } \\
(\mathrm{N}=24)\end{array}$ & $\begin{array}{l}6.5 \text { months } \\
(\mathrm{N}=2)\end{array}$ & - \\
\hline $\begin{array}{l}\text { Etoposide + } \\
\text { ifosfamide }\end{array}$ & Saeter 1995 & 10 & $6(60.0 \%)$ & $0(0.0 \%)$ & $6(60.0 \%)$ & - & - & - & - & - & - & - \\
\hline $\begin{array}{l}\text { Etoposide + } \\
\text { ifosfamide }\end{array}$ & Skubitz 1993 & 15 & $6(40.0 \%)$ & $0(0.0 \%)$ & $6(40.0 \%)$ & - & $5(33.3 \%)$ & - & - & - & $\begin{array}{l}(3.0-31.0+) \sim \\
(N=6)\end{array}$ & $4(26.7 \%)$ \\
\hline $\begin{array}{l}\text { Gemcitabine + } \\
\text { dacarbazine }\end{array}$ & Buesa 2004 & 22 & $\begin{array}{l}5(26.3 \%) \\
(N=19)\end{array}$ & $\begin{array}{l}0(0.0 \%)(\mathrm{N} \\
=19)\end{array}$ & $\begin{array}{l}5(26.3 \%) \\
(N=19)\end{array}$ & - & $\begin{array}{l}6(31.6 \%) \\
(\mathrm{N}=19)\end{array}$ & $\begin{array}{l}3 \text { mo: } 9 \text { (40.9\%) } \\
(\mathrm{N}=22) ; 6 \text { mo: } \\
6(27.3 \%)(\mathrm{N}=22)\end{array}$ & - & - & $\begin{array}{l}6.5 \text { months } \\
(2.5-36.0) \\
(\mathrm{N}=5)\end{array}$ & $\begin{array}{l}8(42.1 \%) \\
(\mathrm{N}=19)\end{array}$ \\
\hline $\begin{array}{l}\text { Gemcitabine + } \\
\text { dacarbazine }\end{array}$ & Losa 2007 & 23 & $1(4.3 \%)$ & $1(4.3 \%)$ & $0(0.0 \%)$ & - & $11(47.8 \%)$ & $\begin{array}{l}3 \text { mo: } 12(46.2 \%) \\
(\mathrm{N}=26) ; 6 \mathrm{mo}: \\
7(26.9 \%)(\mathrm{N}=26)\end{array}$ & $\begin{array}{l}3.6 \text { months } \\
(\mathrm{N}=23)\end{array}$ & $\begin{array}{l}8.5 \text { months } \\
(\mathrm{N}=23)\end{array}$ & - & $11(47.8 \%)$ \\
\hline $\begin{array}{l}\text { Gemcitabine + } \\
\text { docetaxel }\end{array}$ & Hensley 2002 & 16 & $\begin{array}{l}8(50.0 \%) \\
\sim\end{array}$ & $1(6.3 \%) \sim$ & $\begin{array}{l}7(43.8 \%) \\
\sim\end{array}$ & - & - & - & - & - & - & - \\
\hline $\begin{array}{l}\text { Gemcitabine + } \\
\text { docetaxel }\end{array}$ & Hensley 2008 & 48 & $13(27.1 \%)$ & $3(6.3 \%)$ & 10 (20.8\%) & - & $24(50.0 \%)$ & $\begin{array}{l}3 \text { mo: } 35(72.9 \%) \\
(\mathrm{N}=48) ; 6 \mathrm{mo}: \\
25(52.1 \%)(\mathrm{N}=48) ; \\
6.7 \text { months } \\
(0.7-27.0+)(\mathrm{N}=48)\end{array}$ & - & $\begin{array}{l}14.7 \text { months } \\
(0.8-50.9+) \\
(\mathrm{N}=48)\end{array}$ & $\begin{array}{l}9.0 \text { months } \\
(3.9-24.5) \\
(\mathrm{N}=13)\end{array}$ & $8(16.7 \%)$ \\
\hline $\begin{array}{l}\text { Gemcitabine + } \\
\text { docetaxel }\end{array}$ & Montalar 2008 & 12 & 1 (8.3\%)\# & $0(0.0 \%)$ & $1(8.3 \%)$ & - & $4(33.3 \%)$ & - & - & - & - & 7 (58.3\%) \\
\hline $\begin{array}{l}\text { Methotrexate + } \\
\text { vincristine }\end{array}$ & Vaughn 1984 & 14 & $2(14.3 \%)$ & $0(0.0 \%)$ & $2(14.3 \%)$ & - & $3(21.4 \%)$ & - & - & - & - & - \\
\hline$V A C+I E$ & Palumbo 1998 & 12 & $3(25.0 \%)$ & $0(0.0 \%)$ & $3(25.0 \%)$ & - & $4(33.3 \%)$ & - & - & - & - & $3(25.0 \%)$ \\
\hline $\begin{array}{l}C+V C+D+ \\
D T I C+I L-2\end{array}$ & Gravis 2001 & 1 & $0(0.0 \%)$ & $0(0.0 \%)$ & $0(0.0 \%)$ & - & - & - & - & - & - & $1(100 \%)$ \\
\hline$D+I+D T I C+I L-2$ & Gravis 2001 & 9 & $0(0.0 \%)$ & $0(0.0 \%)$ & $0(0.0 \%)$ & - & $1(11.1 \%)$ & - & - & - & - & 8 (88.9\%) \\
\hline$D+I L-2$ & Gravis 2001 & 3 & 1 (33.3\%) & $0(0.0 \%)$ & $1(33.3 \%)$ & - & - & - & - & - & $\begin{array}{l}2.0 \text { months } \\
(\mathrm{N}=1)\end{array}$ & $2(66.7 \%)$ \\
\hline $\begin{array}{l}\text { Carboplatin + } \\
\text { etoposide }\end{array}$ & Holstein 1996 & 8 & 0 (0.0\%)\# & $0(0.0 \%)$ & $0(0.0 \%)$ & - & $2(25.0 \%) \dagger$ & - & - & $\begin{array}{l}12.0 \text { months } \\
(4.0-25.0)\end{array}$ & - & $6(75.0 \%)$ \\
\hline Dacarbazine & Holstein 1996 & 14 & $0(0.0 \%) \#$ & $0(0.0 \%)$ & $0(0.0 \%)$ & - & $2(14.3 \%)+$ & - & - & $\begin{array}{l}5.0 \text { months } \\
(1.0-11.0)\end{array}$ & - & $12(85.7 \%)$ \\
\hline
\end{tabular}

Cl: Confidence Interval; CR: Complete Response; C + VC + D + DTIC + IL-2: Cyclophosphamide + Vincristine + Doxorubicin + Dacarbazine + Interleukin-2; DOR: Duration of Response; D + IL-2: Doxorubicin + Interleukin-2; $\mathrm{D}+\mathrm{I}+\mathrm{DTIC}+$ IL-2: Doxorubicin + Ifosfamide + Dacarbazine + Interleukin-2; INV: Investigator; IRC: Independent Review Committee; mo: Months; MR: Minimal Response; mo: Months; N: Number of evaluable patients; $\mathrm{n}$ : Number with Outcome; ORR: Overall Response Rate; OS: Overall Survival; PFS: Progression-free Survival; PR: Partial Response; SD: Stable Disease; TTP: Time to progression; TRR: Time to Response; VAC + IE: Vincristine + Adriamycin + Cyclophosphamide + Ifosfamide + Etoposide; -Represents data not reported *95\% Confidence Interval; Represents data assesssed by IRC; for other data it was unclear if assessed by investigator or the Independent Review Committee; \#ORR calculated as CR + PR; +Represents no change; \$Represents the subgroup of patients with both RECIST and CHOI evaluations for response. 
Phase II trials is to evaluate if the intervention under investigation demonstrates clinical activity and is well tolerated, and hence, they do not provide a definitive answer regarding the clinical benefit of the intervention in question. Further, post-study therapy was documented in three of the six included RCTs and this may have potentially confounded the OS results [18-20,22-26].

In view of limited RCT evidence, data from nonrandomised studies was evaluated. The 52 prospective non-randomised studies included in the review suggested anti-tumour activity (3-month PFS rate $\geq 39 \%$, and/or 6-month PFS rate $\geq 14 \%$, [90], and/or ORR $\geq 10 \%$ ) of several therapies including: single-agent ifosfamide [77-81] and dacarbazine [73], and that of the combinations, etoposide plus ifosfamide $[86,87]$ and cisplatin plus ifosfamide [85]. Antitumour activity of gemcitabine monotherapy [75] and gemcitabine plus docetaxel $[88,89]$ in patients with uterine leiomyosarcoma was also indicated by the non-RCT evidence. The results observed from the non-randomised evidence should be interpreted in light of the inherent limitations associated with this study design. RCTs involve randomisation which minimises the selection bias and confounding, and are therefore the most rigorous way of determining comparative efficacy.

Despite the systematic approach employed in this review, it was limited by the identification of only a small number of RCTs and the lack of comparability in terms of sample size, study design, and patient populations across both the RCTs and non-randomised studies. The patient population included in the RCT by van Oosterom and colleagues varied from those recruited in other RCTs [29]. The study by van Oosterom and colleagues included a mixed patient population of both first-line and second-line patients, with limited subgroup data for the patients treated in the second-line setting [29]. Most importantly, the RCT evidence was restricted by the fact that there was a lack of head-to-head trials of active agents. Due to the paucity of evidence, indirect and mixed treatment comparison of the included interventions were also not possible as no studies evaluating a common intervention were identified, except the two placebocontrolled trials, wherein an indirect analysis was not feasible due to incompatibility of the data (lack of comparability of the study designs and patient population in these studies) $[18,19,28]$. Although, the review also included 52 prospective non-randomised studies, these studies were small in terms of sample size with majority of studies including less than 50 patients. For several treatments, only single studies were available. When there were multiple studies evaluating a single intervention, variability was often observed in the efficacy and safety results, primarily attributed to differences in patient characteristics and assessment criteria used to evaluate efficacy measures.
With respect to the inclusion criteria of the review, this study was limited to trials evaluating adult patients with sub-types of STS (excluding GIST, Kaposi sarcoma, and Ewing's family of tumours), who had received prior anthracycline and/or ifosfamide therapy for advanced disease. Based on the inclusion criteria of the review, key RCTs including Maki 2007 (gemcitabine vs. gemcitabine plus docetaxel) and Verweij 2000 (docetaxel vs. doxorubicin) and single arm studies including Sleijfer 2009 (pazopanib) and Bay 2006 (gemcitabine plus docetaxel) were excluded as these publications did not provide information fulfilling the inclusion criteria of the review [92-95]. The study by Maki and colleagues included a mixed population of patients treated with zero to three prior chemotherapy regimens with no data specifically reported for patients receiving $\geq 1$ chemotherapy regimen; in addition, the type of prior chemotherapy was unclear [92]. The RCT by Verweij and colleagues was excluded from the review as the study included patients with GIST [93]. In the study by Sleijfer and colleagues, the type of prior therapy was not reported [94], while in the study by Bay and colleagues no sub-group data for patients receiving first-line and later-lines therapies was provided [95]. Additionally, in the study by Bay and colleagues nearly $69 \%$ of patients were treated within the adjuvant setting [95]. A further RCT (TAXOGEM study) investigating gemcitabine vs. gemcitabine plus docetaxel $[27,31]$, whilst identified in our review had not reported efficacy data at the cut-off date for the literature search and thus, while has done subsequently [96], the findings do not contribute to our conclusions.

\section{Clinical perspective}

The primary aim of second and later line treatment of patients with advanced/metastatic soft tissue sarcoma is to delay disease progression and maintain quality of life for as long as possible. The use of an anti-tumour treatment rather than best supportive care should be extensively discussed with the patient and their caregivers. Until now, there has been no standard of care after failure of or intolerance to doxorubicin and/or ifosfamide. An adjusted indirect comparison would be the most appropriate way to compare results of RCTs, but in this case since none of the RCTs had common arms to enable a formal indirect comparison, close attention should be paid to the findings of the individual trials.

In only one trial did the chemotherapy regimen improve overall survival (the combination of gemcitabine plus dacarbazine over dacarbazine alone; Table 2) [20,21]. This trial was not designed to formally demonstrate an overall survival advantage, and therefore, this finding needs to be confirmed by an appropriately designed Phase III trial. Moreover, the sample size of the trial was limited (59 patients received the combination) and there 
is no other published study investigating this original combination $[20,21]$. The full results of the TAXOGEM study [96] are not included in our review for reasons explained earlier but demonstrate the activity of gemcitabine plus docetaxel in patients with leiomyosarcomas and may explain the frequent usage of this combination in clinical practice, especially in those with leiomyosarcomas at uterine sites.

The PALETTE trial has formally demonstrated the benefit of treating patients with anti-angiogenic agent over placebo in terms of PFS in a Phase III setting (Table 2) $[18,19]$. This constitutes a major breakthrough in sarcoma management. However, possibly due to the high usage rate of salvage treatment after progression, this improvement in PFS did not translate into an OS advantage (Table 2).

The every 3 week (q3w) schedule of trabectedin was associated with improvement of PFS, but because of the planned crossover, there was no advantage in term of OS over the weekly schedule (Table 2) [22-26]. Moreover, the weekly schedule may be less convenient than the every 3 week schedule. It should be noted that trabectedin is not currently approved for use in sarcoma in all countries.

Because quality of life and toxicity concerns are of key importance in this setting, the consideration of tolerability and discontinuation rates is as important as efficacy. The traditional cytotoxic drugs commonly induce haematological toxicities whereas grade $3 / 4$ toxicities seen with pazopanib included fatigue, elevated liver enzymes, and hypertension (Table 3). The safety profiles of both approaches (chemotherapy versus pazopanib) appear to be distinct; this is of particular relevance when discussing the toxicity/benefit ratio with patients. Table 4 suggests that discontinuations due to AEs may be more frequent with pazopanib, possibly because oncologists are less familiar with managing the side effects associated with this agent unlike the classical cytotoxic haemotological toxicities, which have been known for years. Discontinuations could also be related to the fact that pazopanib is given continuously unlike cytotoxic therapy, allowing less opportunity for resolution of toxicities.

This review demonstrates that non-randomised trials provide limited information (Table 6). Randomised studies are preferred when designing new trials. The safety profiles of chemotherapy agents versus pazopanib are clearly different, so additional data including compliance, quality of life and cost are needed to fully understand the extent of the differences between chemotherapy and targeted agents.

\section{Conclusions}

Based on this review, the following regimens have demonstrated a PFS advantage: pazopanib over placebo, trabectedin 3-weekly over weekly schedule, and the combination of gemcitabine plus dacarbazine over dacarbazine alone. Consequently, the choice of second- and later-line treatment for advanced STS should consider these interventions. The efficacy/toxicity ratio of therapies which have limited Phase II evidence should be further evaluated in phase III trials based on formal statistical assumptions, and should include parameters such as median overall survival and quality of life.

\section{Additional files}

\section{Additional file 1: PRISMA checklist. This file includes the PRISMA} checklist.

Additional file 2: Search strategy for Embase $^{\circledR}$ and MEDLINE ${ }^{\circledR}$. The file describes the search strategy employed for searching electronic databases Embase $^{\oplus}$ and Medline ${ }^{\circledast}$

Additional file 3: Quality assessment of RCTs. The file describes the quality assessment of RCTs undertaken on the following parameters: Randomisation: was randomisation carried out appropriately? Allocation concealment: Was the concealment of treatment allocation adequate? Baseline comparability: Were the groups similar at the outset of the study in terms of prognostic factors, for example, severity of disease? Blinding: Were the care providers, participants and outcome assessors blind to treatment allocation? Follow-up: Were there any unexpected imbalances in drop-outs between groups? Selective reporting: Is there any evidence to suggest that the authors measured more outcomes than they reported? Analysis: Did the analysis include an intention-to-treat analysis? If so, was this appropriate and were appropriate methods used to account for missing data? Other source of bias: Were there any other sources of bias?

Additional file 4: Key Differences Between Regulatory and Academic Analyses of PALETTE Study. This file highlights the similarities and differences between the two different analyses (regulatory and academic) performed on the PALETTE data.

Additional file 5: Detailed summary of prospective non-randomised studies with sample size more than 10 . This file describes the details of prospective non-randomised studies with sample size more than 10 with respect to study design, sample size, median duration of follow-up, prior therapy, ECOG performance status, and STS sub-type.

Additional file 6: List of prospective small-size studies (sample size $<10$ ) and retrospective studies. This file describes the list of prospective small-size studies (sample size $<10$ ) and retrospective studies included in the review.

Additional file 7: Summary of grade 3 or grade $\mathbf{4}$ specific adverse events observed across non-randomised trials. This file describes the grade 3/4 AEs observed across non-randomised controlled trials.

\section{Abbreviations}

AE: Adverse event; ALT: Alanine aminotransferase; AST: Aspartate aminotransferase; CR: Complete response; DOR: Duration of response; GIST: Gastrointestinal stromal tumour; ORR: Overall response rate; OS: Overall survival; PD: Progressive disease; PFS: Progression-Free survival; PR: Partial response; PRISMA: Preferred reporting items for systematic reviews and meta-analyses; RCTS: Randomised controlled trials; SD: Stable disease; STS: Soft tissue sarcoma; TTP: Time to progression; TTR: Time to response.

\section{Competing interests}

SS and ST are employees of HERON Health Pvt. Ltd., which was commissioned by GSK to undertake the research for this review. SM and SP are employees of GSK. NP declares no competing interests.

\section{Authors' contributions}

SS: participated in the study design, data extraction, article selection, coordination, and manuscript preparation; ST: participated in the article 
selection, data extraction, and manuscript preparation; SM: participated in study design and coordination and helped to draft the manuscript; SP: conceived of the study, and participated in its design and coordination, and helped to draft the manuscript. NP: helped to draft the manuscript. All authors read and approved the final manuscript.

\section{Acknowledgements}

We thank Robert Isbell and Luis Javier Hernandez Pastor who reviewed and provided feedback on the content of the manuscript. This work and the resulting publication have been funded by GSK.

\section{Author details}

${ }^{1}$ Heron Health Pvt. Ltd., Chandigarh, India. ${ }^{2}$ GlaxoSmithKline, Uxbridge, UK.

${ }^{3}$ Department of General Oncology, Centre Oscar Lambret, Lille, France.

Received: 16 July 2012 Accepted: 31 July 2013

Published: 13 August 2013

\section{References}

1. Cormier JN, Pollock RE: Soft tissue sarcomas. CA Cancer J Clin 2004, 54:94-109.

2. Jain A, Sajeevan KV, Babu KG, Lakshmaiah KC: Chemotherapy in adult soft tissue sarcoma. Indian J Cancer 2009, 46:274-287.

3. Weiss SW, Goldblum JR: General considerations. In Enzinger and Weiss's Soft Tissue Tumors. Edited by Weiss SW, Goldblum JR. St Louis, Missouri: CV Mosby; 2001:1-19.

4. Spira Al, Ettinger DS: The use of chemotherapy in soft-tissue sarcomas. Oncologist 2002, 7:348-359.

5. Italiano A, Mathoulin-Pelissier S, Cesne AL, Terrier P, Bonvalot S, Collin F Michels JJ, Blay JY, Coindre JM, Bui B: Trends in survival for patients with metastatic soft-tissue sarcoma. Cancer 2011, 117:1049-1054.

6. Grimer R, Judson I, Peake D, Seddon B: Guidelines for the management of soft tissue sarcomas. Sarcoma 2010, 2010:Article ID: 506182.

7. Casali PG, Blay JY: Soft tissue sarcomas: ESMO Clinical Practice Guidelines for diagnosis, treatment and follow-up. Ann Oncol 2010, 21(Suppl 5):v198-v203.

8. NCCN: NCCN Clinical Practice Guidelines in Oncolcogy: Soft Tissue Sarcoma, Volume V.1.2011. National Comprehensive Cancer Network; 2011. http://www. nccn.org/professionals/physician_gls/pdf/sarcoma.pdf.

9. Leahy M, Garcia DM X, Reichardt P, Judson I, Staddon A, Verweij J, Baffoe-Bonnie A, Jonsson L, Musayev A, Justo N, Burke T, Blay JY: Chemotherapy treatment patterns and clinical outcomes in patients with metastatic soft tissue sarcoma. The SArcoma treatment and Burden of IIIness in North America and Europe (SABINE) study. Ann Oncol 2012: 23(10):2763-70.

10. Liberati A, Altman DG, Tetzlaff J, Mulrow C, Gotzsche PC, loannidis JP, Clarke M, Devereaux PJ, Kleijnen J, Moher D: The PRISMA statement for reporting systematic reviews and meta-analyses of studies that evaluate health care interventions: explanation and elaboration. Ann Intern Med 2009, 151:W65-W94

11. NCCN: NCCN Clinical Practice Guidelines in Oncolcogy: Soft Tissue Sarcoma, Volume V.2.2007. National Comprehensive Cancer Network; 2007. http://www. nccn.org/professionals/physician_gls/pdf/sarcoma.pdf.

12. Verma S, Bramwell V: Dose-intensive chemotherapy in advanced adult soft tissue sarcoma. Expert Rev Anticancer Ther 2002, 2:201-215.

13. Vincenzi B, Frezza AM, Santini D, Tonini G: New therapies in soft tissue sarcoma. Expert Opin Emerg Drugs 2010, 15:237-248.

14. NICE STA template: Specification for manufacturer/sponsor submission of evidence October 2009. 2009. http://www.nice.org.uk/aboutnice/howwework/ devnicetech/singletechnologyappraisalsubmissiontemplates.jsp.

15. The Cochrane Collaboration: Cochrane Handbook for Systematic Reviews of Interventions. 2011. http://www.mrc-bsu.cam.ac.uk/cochrane/handbook/ front_page.htm

16. Downs SH, Black N: The feasibility of creating a checklist for the assessment of the methodological quality both of randomised and non-randomised studies of health care interventions. J Epidemiol Community Health 1998, 52:377-384.

17. Moher D, Liberati A, Tetzlaff J, Altman DG: Preferred reporting items for systematic reviews and meta-analyses: the PRISMA statement. Ann Intern Med 2009, 151:264-269. W64.

18. PALETTE study: A randomized double blind phase III trial of pazopanib versus placebo in patients with soft tissue sarcoma whose disease has progressed during or following prior therapy. 2011. Study summary available at: http://www.gsk-clinicalstudyregister.com/quick-search-list.jsp? item=VEG1 10727\&type=GSK+Study+ID\&studyld=VEG110727.

19. FDA: Votrient ${ }^{\oplus}$ (Pazopanib) Tablets For Treatment of Patients with Soft Tissue Sarcoma: FDA Oncologic Drugs Advisory Committee Briefing Document (NDA 22-465), March 2012. 2012. http://www.fda.gov/ downloads/AdvisoryCommittees/CommitteesMeetingMaterials/Drugs/ OncologicDrugsAdvisoryCommittee/UCM184003.pdf.

20. Garcia-Del-Muro X, Lopez-Pousa A, Maurel J, Martin J, Martinez-Trufero J, Casado A, Gomez-Espana A, Fra J, Cruz J, Poveda A, Meana A, Pericay C, Cubedo R, Rubio J, De JA, Lainez N, Carrasco JA, de AR, Buesa JM: Randomized phase II study comparing gemcitabine plus dacarbazine versus dacarbazine alone in patients with previously treated soft tissue sarcoma: a Spanish Group for Research on Sarcomas study. J Clin Oncol 2011, 29:2528-2533.

21. Garcia Del Muro X, Fra J, Lopez Pousa A, Maurel J, Martin J, Martinez Trufero J, Casado A, Cruz J, Gomez Espana MA, Lavernia J: Randomized phase II study of dacarbazine plus gemcitabine versus DTIC alone in patients with advanced soft tissue sarcoma: A Spanish Group for Research on Sarcomas (GEIS) study. J Clin Oncol 2009, 27:10529.

22. Demetri GD, Chawla SP, von MM, Ritch P, Baker LH, Blay JY, Hande KR, Keohan ML, Samuels BL, Schuetze S, Lebedinsky C, Elsayed YA, Izquierdo MA, Gomez J, Park YC, Le CA: Efficacy and safety of trabectedin in patients with advanced or metastatic liposarcoma or leiomyosarcoma after failure of prior anthracyclines and ifosfamide: results of a randomized phase II study of two different schedules. J Clin Oncol 2009, 27:4188-4196

23. Chawla S, Blay JY, Schuetze S, Morris D, Ritch P, Le Cesne A, Casali PG, Perez J, Bayever E, Demetri G: Efficacy of second-line trabectedin in patients with advanced liposarcomas and leiomyosarcomas progressing despite prior conventional chemotherapy. Eur J Cancer, Supp/ 2009, 7:598.

24. Morgan JA, Le Cesne A, Chawla S, von Mehren M, Schuetze S, Casali PG, Nieto A, Elsayed Y, Izquierdo MA, Demetri GD: Randomized phase II study of trabectedin in patients with liposarcoma and leiomyosarcoma (L-sarcomas) after failure of prior anthracylines (A) and ifosfamide (I). ASCO 2007:Abstract No.: 10060.

25. Demetri GD, Schuetze S, Blay J, Chawla S, von Mehren M, Casali P, Morris D, Bayever $\mathrm{E}$, Alfaro $V$, Le Cesne A: Long-term results of a randomized phase II study of trabectedin by two different dose and schedule regimens in patients with advanced liposarcoma or leiomyosarcoma after failure of prior anthracyclines and ifosfamide. J Clin Oncol 2009, 27:10509.

26. Chawla S, Casali PG, von Mehren M, Le Cesne A, Blay JY, Lebedinsky C, Alfaro $V$, Elsayed $Y$, Michiels B, Demetri GD: Clinical tolerability of trabectedin administered by two different schedule (weekly for 3 of 4 weeks vs. q3 weeks) in patients with advanced/metastatic liposarcoma or leiomyosarcoma (L-sarcomas) progressing despite prior treatment with at least anthracycline and ifosfamide. Abstract at European Cancer Organisation (ECCO) Ann Meet Eur J Cancer 2007, 5:Abstract No.: 7517.

27. Pautier P, Bui Nguyen B, Penel N, Piperno-Neumann S, Delcambre-Lair C, Bompas E, Collin F, Rey A, Jimenez M, Duffaud F: Final results of a FNCLCC French Sarcoma Group multicenter randomized phase II study of gemcitabine $(G)$ versus gemcitabine and docetaxel $(G+D)$ in patients with metastatic or relapsed leiomyosarcoma (LMS). J Clin Oncol 2009, 27:10527.

28. Pacey S, Ratain MJ, Flaherty KT, Kaye SB, Cupit L, Rowinsky EK, Xia C, O'Dwyer PJ, Judson IR: Efficacy and safety of sorafenib in a subset of patients with advanced soft tissue sarcoma from a Phase II randomized discontinuation trial. Invest New Drugs 2011, 29:481-488.

29. van Oosterom AT, Mouridsen HT, Nielsen OS, Dombernowsky P, Krzemieniecki K, Judson I, Svancarova L, Spooner D, Hermans C, Van GM, Verweij J: Results of randomised studies of the EORTC Soft Tissue and Bone Sarcoma Group (STBSG) with two different ifosfamide regimens in first- and second-line chemotherapy in advanced soft tissue sarcoma patients. Eur J Cancer 2002, 38:2397-2406.

30. Van Der Graaf WT, Blay J, Chawla SP, Kim D, Bui Nguyen B, Casali PG, Schoffski P, Aglietta M, Staddon AP, Beppu Y, Le Cesne A, Gelderblom H, Judson IR, Araki N, Ouali M, Marreaud S, Hodge R, Dewji M, Dei Tos AP, Hohenberge, PALETTE: A randomized, double-blind, phase III trial of pazopanib versus placebo in patients (pts) with soft-tissue sarcoma (STS) whose disease has progressed during or following prior chemotherapy An EORTC STBSG Global Network Study (EORTC 62072). Abstract and 
oral presentation at the American Society of Clinical Oncology Annual Meeting 2011. J Clin Oncol 2011, 29:Abstract no. LBA10002.

31. Duffaud F, Bui BN, Penel N, Cioffi A, Isambert N, Blay JY, Cupissol D, Jimenez M, Rey A, Pautier P: A FNCLCC French Sarcoma Group--GETO multicenter randomized phase II study of gemcitabine (G) versus gemcitabine and docetaxel $(\mathrm{G}+\mathrm{D})$ in patients with metastatic or relapsed leiomyosarcoma (LMS). Abstract American Society of Oncology (ASCO) Ann Meet J Clin Oncol 2008, 26:Abstract No.: 10511

32. Van Der Graaf WT, Blay JY, Chawla SP, Kim DW, Bui-Nguyen B, Casali PG, Schoffski P, Aglietta M, Staddon AP, Beppu Y, Le CA, Gelderblom H, Judson IR, Araki N, Ouali M, Marreaud S, Hodge R, Dewji MR, Coens C, Demetri GD, Fletcher CD, Dei Tos AP, Hohenberger P: Pazopanib for metastatic soft-tissue sarcoma (PALETTE): a randomised, double-blind, placebo-controlled phase 3 trial. Lancet 2012, 379:1879-1886.

33. Leahy M, Ray-Coquard I, Verweij J, Le CA, Duffaud F, Hogendoorn PC, Fowst C, de BC, di Paola ED, Van GM, Judson I, Blay JY: Brostallicin, an agent with potential activity in metastatic soft tissue sarcoma: A phase II study from the European Organisation for Research and Treatment of Cancer Soft Tissue and Bone Sarcoma Group. Eur J Cancer 2007, 43:308-315.

34. Thigpen JT, Blessing JA, Wilbanks GD: Cisplatin as second-line chemotherapy in the treatment of advanced or recurrent leiomyosarcoma of the uterus. A Phase II trial of the Gynecologic Oncology Group. Am J Clin Oncol: Cancer Clin Trials 1986, 9:18-20.

35. Bramwell VHC, Mouridsen HT, Santoro A, Blackledge G, Somers R, Verweij J, Dombernowsky P, Onsrud M, Thomas D, Sylvester R, van Oosterom A, Suppl 2: Cyclophosphamide versus ifosfamide: a randomized phase II trial in adult soft-tissue sarcomas. The European Organization for Research and Treatment of Cancer [EORTC], Soft Tissue and Bone Sarcoma Group. Cancer Chemother Pharmacol 1993, 31:S180-S184.

36. Bramwell VHC, Mouridsen HT, Santoro A, Blackledge G, Somers R, Verweij J, Dombernowsky P, Onsrud M, Thomas D, Sylvester R, van Oosterom A: Cyclophosphamide versus ifosfamide: Final report of a randomized phase II trial in adult soft tissue sarcomas. Eur J Cancer Clin Oncol 1987, 23:311-321.

37. Kostler WJ, Brodowicz T, Attems Y, Hejna M, Tomek S, Amann G, Fiebiger WC, Wiltschke CH, Krainer M, Zielinski CC: Docetaxel as rescue medication in anthracycline- and ifostamide-resistant locally advanced or metastatic soft tissue sarcoma: Results of a phase II trial. Ann Oncol 2001, 12:1281-1288.

38. Santoro A, Romanini A, Rosso A, Frustaci S, Comandone A, Apice G, De TD, Dogliotti L, Lionetto R, Dani C, Bruzzi P, Piolini M, Bergnolo P, Verusio C: Lack of activity of docetaxel in soft tissue sarcomas: Results of a phase II study of the Italian Group on Rare Tumors. Sarcoma 1999, 3:177-181.

39. Van Hoesel QG, Verweij J, Catimel G, Clavel M, Kerbrat P, Van Oosterom AT, Kerger J, Tursz T, Van GM, van PC: Phase II study with docetaxel (Taxotere ${ }^{\oplus}$ ) in advanced soft tissue sarcomas of the adult. Ann Oncol 1994, 5:539-542.

40. Verweij J: Docetaxel: An interesting new drug for the treatment of head and neck cancers and soft tissue sarcomas. Anti-Cancer Drugs 1995, 6:19-24.

41. Mouridsen HT, Bastholt L, Somers R, Santoro A, Bramwell V, Mulder JH, van Oosterom AT, Buesa J, Pinedo HM, Thomas D: Adriamycin versus epirubicin in advanced soft tissue sarcomas. A randomized phase II/phase III study of the EORTC Soft Tissue and Bone Sarcoma Group. E J Cancer Clin Oncol 1987, 23:1477-1483.

42. Crawley CR, Judson IR, Verrill M, Hill C, Raynaud FI: A phase I/II study of a 72-h continuous infusion of etoposide in advanced soft tissue sarcoma. Sarcoma 1997, 1:149-154.

43. Dombernowsky P, Buesa J, Pinedo HM, Santoro A, Mouridsen H, Somers R, Bramwell V, Onsrud M, Rouesse J, Thomas D: VP-16 in advanced soft tissue sarcoma: A phase II study of the EORTC soft tissue and bone sarcoma group. Eur J Cancer Clin Oncol 1987, 23:579-580.

44. Ray-Coquard I, Le CA, Whelan JS, Schoffski P, Bui BN, Verweij J, Marreaud S, Van GM, Hogendoorn P, Blay JY: A phase II study of gefitinib for patients with advanced HER-1 expressing synovial sarcoma refractory to doxorubicin-containing regimens. Oncologist 2008, 13:467-473.

45. Hartmann JT, Oechsle K, Huober J, Jakob A, Azemar M, Horger M, Kanz L, Bokemeyer C: An open label, non-comparative phase II study of gemcitabine as salvage treatment for patients with pretreated adult type soft tissue sarcoma. Invest New Drugs 2006, 24:249-253.

46. Spath-Schwalbe E, Genvresse I, Koschuth A, Dietzmann A, Grunewald R, Possinger K: Phase II trial of gemcitabine in patients with pretreated advanced soft tissue sarcomas. Anti -Cancer Drugs 2000, 11:325-329.
47. Antman KH, Montella D, Rosenbaum C, Schwen M: Phase II trial of ifosfamide with mesna in previously treated metastatic sarcoma. Cancer Treat Rep 1985, 69:499-504.

48. Antman KH, Ryan L, Elias A, Sherman D, Grier HE: Response to ifosfamide and mesna: 124 previously treated patients with metastatic or unresectable sarcoma. J Clin Oncol 1989, 7:126-131.

49. Tursz T: High-dose ifosfamide in the treatment of advanced soft tissue sarcomas. Semin Oncol 1996, 23:34-39.

50. Skubitz KM: Phase II trial of pegylated-liposomal doxorubicin (Doxil (trademark)) in sarcoma. Cancer Invest 2003, 21:167-176.

51. Toma S, Tucci A, Villani G, Carteni G, Spadini N, Palumbo R: Liposomal doxorubicin (Caelyx) in advanced pretreated soft tissue sarcomas: A phase II study of the Italian Sarcoma Group (ISG). Anticancer Res 2000, 20:485-491.

52. Buesa JM, Mouridsen HT, Santoro A, Somers R, Bramwell V, van Oosterom AT, Wagener T, Vendrik C, Thomas D: Treatment of advanced soft tissue sarcomas with low-dose methotrexate: A phase II trial by the European Organization for Research on Treatment of Cancer (EORTC) soft tissue and bone sarcoma group. Cancer Treat Rep 1984, 68:683-684.

53. Palumbo R, Raffo P, Capello C, Castagneto B, Gatti C, Toma S: Paclitaxel (taxol) in pretreated, relapsed and/or metastatic adult soft tissue sarcomas (STS). Oncol Rep 1997, 4:127-130.

54. Patel SR, Linke KA, Burgess MA, Papadopoulos NE, Plager C, Jenkins J, Benjamin R: Phase II study of paclitaxel in patients with soft tissue sarcomas. Sarcoma 1997, 1:95-97.

55. Skubitz KM: A phase I study of ambulatory continuous infusion paclitaxel. Anti-Cancer Drugs 1997, 8:823-828.

56. Bertuzzi A: Efficacy and toxicity of sorafenib monotherapy in patients with advanced soft tissue sarcoma failing anthracycline-based chemotherapy. J Clin Oncol 2010, 28:Abstract No.: 10025.

57. Bertuzzi A, Stroppa E, Secondino S, Zucali P, Quagliuolo V, Pedrazzoli P, Comandone A, Basso U, Soto Parra H, Santoro A: Efficacy and toxicity of sorafenib in patients with advanced soft tissue sarcoma failing anthracycline-based chemotherapy. Eur J Cancer, Supp/ 2009, 7:599.

58. Decoster LV, Vande Broek I, Anckaert E, De Mey J, Denys H, Canon J, De Clerck D, Neyns B, De Greve J: Activity of sunitinib in advanced soft tissue sarcoma and its correlation with potential predictive biomarkers. Ann Oncol 2010, 21:viii413.

59. Schoffski P, Wolter P, Clement P, Sciot R, De WI, Wozniak A, Stefan C, Dumez H: Trabectedin (ET-743): Evaluation of its use in advanced soft-tissue sarcoma. Future Oncol 2007, 3:381-392.

60. Demetri GD: ET-743: The US experience in sarcomas of soft tissues. Anti -Cancer Drugs 2002, 13:S7-S9.

61. Brain EGC: Safety and efficacy of ET-743: The French experience. Anti-Cancer Drugs 2002, 13:S11-S14.

62. Bramwell VHC, Morris D, Ernst DS, Hings I, Blackstein M, Venner PM, Ette El, Harding MW, Waxman A, Demetri GD: Safety and efficacy of the multidrug-resistance inhibitor biricodar (VX-710) with concurrent doxorubicin in patients with anthracycline-resistant advanced soft tissue sarcoma. Clin Cancer Res 2002, 8:383-393.

63. Keohan ML, Grever MR, Balcerzak SP, Antman K: A phase II Southwest Oncology Group study of cisplatin and continuous infusion vinblastine in the treatment of advanced soft tissue sarcoma. Invest New Drugs 1997, 15:255-256.

64. Le Cesne A, Vassal G, Farace F, Spielmann M, Le CT, Angevin E, Valteau-Couanet D, Fizazi K, Cojean I, Llombard A, Tursz T, Escudier B: Combination interleukin-2 and doxorubicin in advanced adult solid tumors: Circumvention of doxorubicin resistance in soft-tissue sarcoma? J Immunother 1999, 22:268-277.

65. Lopez M, Carpano S, Dilauro L, Chiatti L, Vici P, Cavaliere F, Gentile P, Citro G: Clinical modulation of epirubicin resistance by lonidamine in patients with advanced soft-tissue sarcomas. Int J Oncol 1995, 6:363-367.

66. Buesa JM, Losa R, Fernandez A, Sierra M, Esteban E, Diaz A, Lopez-Pousa A, Fra J: Phase I clinical trial of fixed-dose rate infusional gemcitabine and dacarbazine in the treatment of advanced soft tissue sarcoma, with assessment of gemcitabine triphosphate accumulation. Cancer 2004, 101:2261-2269.

67. Losa R, Fra J, Lopez-Pousa A, Sierra M, Goitia A, Una E, Nadal R, Del Muro JG, Gion M, Maurel J, Escudero P, Esteban E, Buesa JM: Phase II study with the combination of gemcitabine and DTIC in patients with advanced soft tissue sarcomas. Cancer Chemother Pharmacol 2007, 59:251-259.

68. Montalar J, Diaz R, Santaballa A, de la Cueva H, Richart P, Cortbellas M, Garcia J, Segura Huerta AA, Aparisi F: Second-line chemotherapy with gemcitabine-docetaxel in patients with advanced soft-tissue sarcomas 
after treament with high-dose ifosfamide and adriamycin : A single-centre experience. Ann Oncol 2008, 19:viii270.

69. Vaughn CB, McKelvey E, Balcerzak SP, Loh K, Stephens R, Baker L: High-dose methotrexate with leucovorin rescue plus vincristine in advanced sarcoma: A Southwest Oncology Group study. Cancer Treat Rep 1984, 68:409-410.

70. Palumbo R, Palmeri S, Gatti C, Villani G, Cesca A, Toma S: Combination chemotherapy using vincristine, adriamycin, cyclophosphamide (VAC) alternating with ifosfamide and etoposide (IE) for advanced soft tissue sarcomas: A phase II study. Oncol Rep 1998, 5:69-72.

71. Gravis G, Mousseau M, Douillard JY, Dorval T, Fabbro M, Escudier B, Mignot L, Viens P: Can interleukin-2 reverse anthracyclin chemoresistance in metastatic soft tissue sarcoma patients. Results of a prospective phase II clinical trial. Eur Cytokine Netw 2001, 12:239-243.

72. Holstein K, Welt HJ, Walter TA, Llossfeld DK: Salvage chemotherapy with dacarbazine or carboplatin/VP-16 of advanced soft tissue sarcoma pretreated with doxorubicin/ifosfamide. Onkologie 1996, 19:496-499.

73. Buesa JM, Mouridsen HT, van Oosterom AT, Verweij J, Wagener T, Steward W, Poveda A, Vestlev PM, Thomas D, Sylvester R: High-dose DTIC in advanced soft-tissue sarcomas in the adult. Ann Oncol 1991, 2:307-309.

74. Ferraresi V, Ciccarese M, Cercato MC, Nuzzo C, Zeuli M, Di FF, Giannarelli D, Cognetti F: Gemcitabine at fixed dose-rate in patients with advanced soft tissue sarcomas: A mono-institutional phase II study. Cancer Chemother Pharmacol 2008, 63:149-155.

75. Look KY, Sandler A, Blessing JA, Lucci JA III, Rose PG: Phase II trial of gemcitabine as second-line chemotherapy of uterine leiomyosarcoma: A Gynecologic Oncology Group (GOG) Study. Gynecol Oncol 2004, 92:644-647.

76. Babovic N, Jelic S, Milanovic N, Matkovic S: Pilot study of daily ifosfamide $1 \mathrm{~g} / \mathrm{m} 2$ until grade III granulocytopenia as second-line chemotherapy for anthracyclinepretreated advanced soft tissue sarcoma. Tumori 1998, 84:677-680.

77. Le Cesne A, Antoine E, Spielmann M, Le CT, Brain E, Toussaint C, Janin N, Kayitalire L, Fontaine F, Genin J: High-dose ifosfamide: circumvention of resistance to standard-dose ifosfamide in advanced soft tissue sarcomas. J Clin Oncol 1995, 13:1600-1608.

78. Nielsen OS, Judson I, van HQ, Le CA, Keizer HJ, Blay JY, van OA, Radford JA Svancarova L, Krzemienlecki K, Hermans C, Van GM, Oosterhuis JW, Verweij J: Effect of high-dose ifosfamide in advanced soft tissue sarcomas. A multicentre phase II study of the EORTC Soft Tissue and Bone Sarcoma Group. Eur J Cancer 2000, 36:61-67.

79. Palumbo R, Palmeri S, Antimi M, Gatti C, Raffo P, Villani G, Toma S: Phase II study of continuous-infusion high-dose ifosfamide in advanced and/or metastatic pretreated soft tissue sarcomas. Ann Oncol 1997, 8:1159-1162.

80. Patel SR, Vadhan-Raj S, Papadopolous N, Plager C, Burgess MA, Hays C, Benjamin RS: High-dose ifosfamide in bone and soft tissue sarcomas: Results of phase II and pilot studies - Dose-response and schedule dependence. J Clin Oncol 1997, 15:2378-2384.

81. Scheulen ME, Niederle N, Bremer K, Schutte J, Seeber S: Efficacy of ifosfamide in refractory malignant diseases and uroprotection by mesna: Results of a clinical phase II-study with 151 patients. Cancer Treat Rev 1983, 10:93-101.

82. Garcia-Carbonero R, Supko JG, Manola J, Seiden MV, Harmon D, Ryan DP, Quigley MT, Merriam P, Canniff J, Goss G, Matulonis U, Maki RG, Lopez T, Puchalski TA, Sancho MA, Gomez J, Guzman C, Jimeno J, Demetri GD: Phase II and pharmacokinetic study of ecteinascidin 743 in patients with progressive sarcomas of soft tissues refractory to chemotherapy. J Clin Oncol 2004, 22:1480-1490.

83. Le Cesne A, Blay JY, Judson I, van OA, Verweij J, Radford J, Lorigan P Rodenhuis S, Ray-Coquard I, Bonvalot S, Collin F, Jimeno J, Di PE, Van GM, Nielsen OS: Phase II study of ET-743 in advanced soft tissue sarcomas: A European Organisation for the Research and Treatment of Cancer (EORTC) Soft Tissue and Bone Sarcoma Group trial. J Clin Oncol 2005, 23:576-584

84. Yovine A, Riofrio M, Blay JY, Brain E, Alexandre J, Kahatt C, Taamma A, Jimeno J, Martin C, Salhi Y, Cvitkovic E, Misset JL: Phase II study of ecteinascidin-743 in advanced pretreated soft tissue sarcoma patients. J Clin Oncol 2004, 22:890-899.

85. Budd GT, Metch B, Weiss SA, Weick JK, Fabian C, Stephens RL, Balcerzak SP: Phase II trial of ifosfamide and cisplatin in the treatment of metastatic sarcomas: A Southwest Oncology Group study. Cancer Chemother Pharmacol 1993, 31:S213-S216.
86. Saeter G, Talle K, Solheim OP: Treatment of advanced, high-grade softtissue sarcoma with ifosfamide and continuous-infusion etoposide. Cancer Chemother Pharmacol 1995, 36:172-175.

87. Skubitz KM, Hamdan H, Thompson RC Jr: Ambulatory continuous infusion ifosfamide with oral etoposide in advanced sarcomas. Cancer 1993, 72:2963-2969

88. Hensley ML, Maki R, Venkatraman E, Geller G, Lovegren M, Aghajanian C, Sabbatini P, Tong W, Barakat R, Spriggs DR: Gemcitabine and docetaxel in patients with unresectable leiomyosarcoma: Results of a phase II trial. J Clin Oncol 2002, 20:2824-2831.

89. Hensley ML, Blessing JA, Degeest K, Abulafia O, Rose PG, Homesley HD: Fixed-dose rate gemcitabine plus docetaxel as second-line therapy for metastatic uterine leiomyosarcoma: A Gynecologic Oncology Group phase II study. Gynecol Oncol 2008, 109:323-328.

90. Van Glabbeke M, Verweij J, Judson I, Nielsen OS: Progression-free rate as the principal end-point for phase II trials in soft-tissue sarcomas. Eur J Cancer 2002, 38:543-549.

91. Penel N, Van GM, Marreaud S, Ouali M, Blay JY, Hohenberger P: Testing new regimens in patients with advanced soft tissue sarcoma: analysis of publications from the last 10 years. Ann Oncol 2011, 22:1266-1272.

92. Maki RG, Wathen JK, Patel SR, Priebat DA, Okuno SH, Samuels B, Fanucchi M, Harmon DC, Schuetze SM, Reinke D, Thall PF, Benjamin RS, Baker LH, Hensley ML: Randomized phase II study of gemcitabine and docetaxel compared with gemcitabine alone in patients with metastatic soft tissue sarcomas: results of sarcoma alliance for research through collaboration study 002 [corrected]. J Clin Oncol 2007, 25:2755-2763.

93. Verweij J, Lee SM, Ruka W, Buesa J, Coleman R, van HR, Seynaeve C, di Paola ED, Van GM, Tonelli D, Judson IR: Randomized phase II study of docetaxel versus doxorubicin in first- and second-line chemotherapy for locally advanced or metastatic soft tissue sarcomas in adults: a study of the european organization for research and treatment of cancer soft tissue and bone sarcoma group. J Clin Oncol 2000, 18:2081-2086.

94. Sleijfer S, Ray-Coquard I, Papai Z, Le CA, Scurr M, Schoffski P, Collin F, Pandite L, Marreaud S, De BA, Van GM, Verweij J, Blay JY: Pazopanib, a multikinase angiogenesis inhibitor, in patients with relapsed or refractory advanced soft tissue sarcoma: a phase II study from the European organisation for research and treatment of cancer-soft tissue and bone sarcoma group (EORTC study 62043). J Clin Oncol 2009, 27:3126-3132.

95. Bay JO, Ray-Coquard I, Fayette J, Leyvraz S, Cherix S, Piperno-Neumann S, Chevreau C, Isambert N, Brain E, Emile G, Le CA, Cioffi A, Kwiatkowski F, Coindre JM, Bui NB, Peyrade F, Penel N, Blay JY: Docetaxel and gemcitabine combination in 133 advanced soft-tissue sarcomas: a retrospective analysis. Int J Cancer 2006, 119:706-711.

96. Pautier P, Floquet A, Penel N, Piperno-Neumann S, Isambert N, Rey A, Bompas E, Cioffi A, Delcambre C, Cupissol D, Collin F, Blay JY, Jimenez M, Duffaud F: Randomized multicenter and stratified phase II study of gemcitabine alone versus gemcitabine and docetaxel in patients with metastatic or relapsed leiomyosarcomas: a Federation Nationale des Centres de Lutte Contre le Cancer (FNCLCC) French Sarcoma Group Study (TAXOGEM study). Oncologist 2012, 17:1213-1220.

doi:10.1186/1471-2407-13-385

Cite this article as: Sharma et al:: Efficacy and safety of pharmacological interventions in second- or later-line treatment of patients with advanced soft tissue sarcoma: a systematic review. BMC Cancer 2013 13:385 